A resolution of Stewartson's quarter-infinite plate problem

Duck, P.W. and Hewitt, R.E.

2012

MIMS EPrint: 2013.71

Manchester Institute for Mathematical Sciences

School of Mathematics

The University of Manchester

\footnotetext{
Reports available from: http://eprints.maths.manchester.ac.uk/

And by contacting: The MIMS Secretary

School of Mathematics

The University of Manchester

Manchester, M13 9PL, UK
} 


\title{
A resolution of Stewartson's quarter-infinite plate problem
}

Received: 23 September 2010 / Accepted: 22 February 2011 / Published online: 10 March 2011

(C) Springer-Verlag 2011

\begin{abstract}
We revisit a problem originally considered by Stewartson in 1961: the incompressible, high-Reynolds-number flow past a quarter-infinite plate, with a leading edge that is perpendicular to, and a side edge that is parallel to, an undisturbed oncoming freestream. Particular emphasis is placed on the key region close to the side edge, where the flow is (superficially) three-dimensional, although the use of similarity variables reduces the dimensionality of the problem down to two. As noted by Stewartson, this problem has several intriguing features; it includes singularities and is also of a mixed parabolic type, with edge conditions influencing the solution in both directions across the flow domain. These features serve to greatly complicate the (numerical) solution process (the problem is of course also highly non-linear), and computation was clearly infeasible in 1961. In the present paper, a detailed computational study is presented, answering many of the questions that arose from the 1961 study. We present detailed numerical results together with asymptotic analyses of the key locations in the flow.
\end{abstract}

Keywords Boundary-layer · Mixed-parabolic · Quarter plate

\section{Introduction}

Although the application of asymptotic techniques to high-Reynolds-number flows can be traced back many years, for example to [4] (and arguably even back to [1]), it was not until the development of more formal (and rigorous) asymptotic techniques (including that of matched asymptotic expansions) that the full power of such techniques was realised. Stewartson was at the vanguard of the use of formal asymptotic methods, which for the triple-deck problem resolved the nature of high-Reynolds-number flow in the vicinity of the trailing edge of a flat plate; see for example [6,13] and [7]. These asymptotic methods were further developed for, and gave an enlightened view of, flow separation (see [15] for a review of early work in this area), flow instabilities and even early-stage transition (e.g. [3]). The methods were also made popular to a more general applied mathematics (and engineering) readership, through the publication of a number of excellent texts, including [17].

In one of Stewartson's early works on high-Reynolds-number flows ([12], hereafter referred to as S61), he studied the high-Reynolds-number flow past a quarter-infinite plate, i.e. a plate with both a leading and a

Communicated by: M. Y. Hussaini.

P. W. Duck $(\bowtie) \cdot$ R. E. Hewitt

School of Mathematics, University of Manchester, Manchester, M13 9PL, UK

E-mail: duck@ma.man.ac.uk

R. E. Hewitt

E-mail: richard.e.hewitt@manchester.ac.uk 
side edge. Ahead of this plate, the flow is perpendicular to the leading edge, but parallel to the side edge (The genesis for this study can be found in the work of [5].).

At locations on the plate, but sufficiently far from the side edge, the flow is expected to be effectively Blasius-like. However, the region of real interest in this problem is where side-edge effects become important: this is the problem first considered in S61 and is revisited in the present paper. Given the relative immature development of asymptotic analysis in the early 1960s (especially matched asymptotic analysis), the discussion presented in S61 is a tour de force, as exemplified by the presence of logarithmically small terms, which are properly taken account of.

In S61, it was shown that the problem in the key side-edge region could be reduced to two dimensions through the use of similarity variables. It should be emphasised these transformations are somewhat different from what a naïve guess would suggest, and involve the presence of the aforementioned logarithmic terms. The key result from S61 was the derivation of two highly non-linear, third-order, parabolic partial differential equations. However, changes in the sign of a key term lead to changes in the directionality of the parabolic system, in turn leading to the bidirectional influence of far-field boundary conditions both on and off the plate. The sign of this crucial term (denoted by $\kappa$ in S61 and defined later herein) indicates the direction of disturbance propagation in the flow domain, a feature that is discussed in S61, page 8. This bidirectionality, together with discontinuous boundary conditions, serves to make this a very challenging system to compute, even by today's standards. Obviously, in 1961, computational hardware and techniques were very much in their infancy, and Stewartson could only speculate as to some of the characteristics of the solution of this system. We will clarify some of the speculative comments in the light of our numerical and asymptotic investigations.

The layout of the paper is as follows: in Sect. 2, we present a formulation of the problem, where we endeavour to be faithful to the notation of S61. In Sect. 3, we discuss the numerical problem (based on a directsolver algorithm), together with a selection of numerical results. In Sect. 4, a number of asymptotic results are presented, which give added insight into (and confidence in) our numerical results. Section 5 presents a final discussion.

\section{Formulation}

We consider a steady, viscous, incompressible, three-dimensional flow, and so our starting point is the appropriate form of the Navier-Stokes equations, together with continuity, namely

$$
\begin{aligned}
& u \frac{\partial u}{\partial x}+v \frac{\partial u}{\partial y}+w \frac{\partial u}{\partial z}=-\frac{1}{\rho} \frac{\partial p}{\partial x}+v\left(\frac{\partial^{2} u}{\partial x^{2}}+\frac{\partial^{2} u}{\partial y^{2}}+\frac{\partial^{2} u}{\partial z^{2}}\right), \\
& u \frac{\partial v}{\partial x}+v \frac{\partial v}{\partial y}+w \frac{\partial v}{\partial z}=-\frac{1}{\rho} \frac{\partial p}{\partial y}+v\left(\frac{\partial^{2} v}{\partial x^{2}}+\frac{\partial^{2} v}{\partial y^{2}}+\frac{\partial^{2} v}{\partial z^{2}}\right), \\
& u \frac{\partial w}{\partial x}+v \frac{\partial w}{\partial y}+w \frac{\partial w}{\partial z}=-\frac{1}{\rho} \frac{\partial p}{\partial z}+v\left(\frac{\partial^{2} w}{\partial x^{2}}+\frac{\partial^{2} w}{\partial y^{2}}+\frac{\partial^{2} w}{\partial z^{2}}\right), \\
& \frac{\partial u}{\partial x}+\frac{\partial v}{\partial y}+\frac{\partial w}{\partial z}=0 .
\end{aligned}
$$

Here, the velocity components $(u, v, w)$ are in the Cartesian $(x, y, z)$ directions, respectively, the pressure is $p, \rho$ denotes the constant density, and $v$ the kinematic viscosity of the fluid. The quarter plate lies in the domain $y=0, x>0, z>0$, on which no slip $(u=v=w=0)$ is enforced, otherwise symmetry in $u$ and $w$ and antisymmetry in $v$ apply across $y=0$. The undisturbed velocity vector is $U(1,0,0)$, where $U$ denotes the velocity scale in the freestream (and indeed will serve to non-dimensionalise velocities throughout) see Fig. 1.

Outside any boundary/shear layers, the flow must be potential, and so the velocity vector can be written

$$
(u, v, w)=(U, 0,0)+\nabla \Phi,
$$

where $\Phi$ is harmonic, $\nabla \Phi \rightarrow 0$ at locations remote from the plate, and

$$
\left.\frac{\partial \Phi}{\partial y}\right|_{y=0^{+}}=\left\{\begin{array}{lc}
\delta^{*} U(v / x U)^{\frac{1}{2}}, & x>0, z>0 \\
0, & \text { otherwise }
\end{array},\right.
$$

where $\delta^{*} \approx 0.860$ (the displacement thickness from the Blasius solution). 


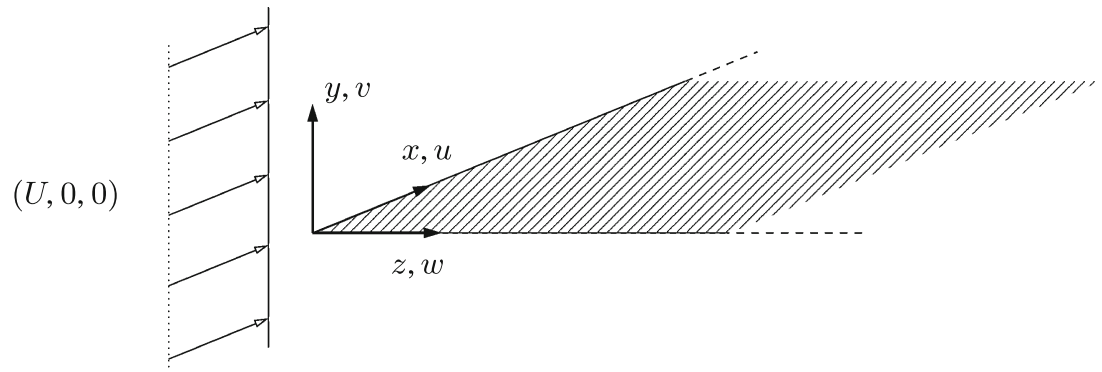

Fig. 1 A schematic representation of the flow geometry; a Cartesian coordinate system $(x, y, z)$ with velocity field $(u, v, w)$ is centred at the corner of the plate. The plate exists on $y=0$, with the leading edge at $x=0$ and side edge at $z=0$; the (uniform) freestream flow is aligned with the side edge with uniform speed $U$

Following [5] and S61, the solution for $\Phi$ can be written in the form

$$
\Phi(x, y, z)=-\frac{\delta^{*}}{2 \pi}\left(\frac{U v}{\pi}\right)^{\frac{1}{2}} \int_{0}^{\infty} \int_{0}^{\infty} \frac{\mathrm{d} \bar{x} \mathrm{~d} \bar{z}}{\bar{x}^{\frac{1}{2}}\left\{(x-\bar{x})^{2}+y^{2}+(z-\bar{z})^{2}\right\}^{\frac{1}{2}}} .
$$

In S61, the regimes $z / x \gg 1$ and $|z / x| \ll 1$ were considered. The former case corresponds to the regime where the flow is predominantly Blasius-like and was fully considered in S61. The analysis of S61 also mentions the region in the immediate vicinity of the leading edge; this was a problem later considered and solved by [2] for the two-dimensional case and is a region governed by the full Navier-Stokes equations.

Here, we focus our attention on the key region near the side edge. Following S61, we write $\xi=z / x$, and then if this quantity is small and as $y \rightarrow 0$, from (4)

$$
\begin{aligned}
& \frac{\partial \Phi}{\partial x} \sim-\frac{\delta^{*} U \xi}{2}(v / x U)^{\frac{1}{2}}\left(\log \frac{\xi}{8}+1\right), \\
& \frac{\partial \Phi}{\partial z} \sim \frac{\delta^{*} U}{\pi}(v / x U)^{\frac{1}{2}} \log \frac{\xi}{8} .
\end{aligned}
$$

It is the occurrence of the logarithmic terms above that serves to somewhat complicate the overall picture in the boundary layer, especially in the crossflow (z) direction. A plausible, but naïve suggestion would be to introduce two inner similarity variables based on

$$
\eta=y\left(\frac{U}{2 v x}\right)^{\frac{1}{2}}, \quad \theta=z\left(\frac{U}{2 v x}\right)^{\frac{1}{2}},
$$

but the latter variable is correctly dismissed in S61, basically on the grounds of being unable to satisfy the continuity equation in any meaningful way. Whilst the $\eta$ similarity variable defined above is entirely appropriate, S61 determined that the appropriate crossflow similarity variable is not $\theta$ but rather

$$
\zeta=\frac{2 \pi}{\delta^{*}}\left(\frac{U}{v x}\right)^{\frac{1}{2}} \frac{z}{\log (U x / v)}
$$

This represents a crossflow scale somewhat longer than that represented by the $\theta$ scale defined in (6) above, due to the occurrence of the logarithmic term in the denominator (note that the high-Reynolds-number assumption implies that $U x / v \gg 1$ ).

We continue to follow the path described in S61, by the introduction of the following two variables $X, Z$, which enables the continuity equation to be automatically satisfied:

$$
\begin{aligned}
u & =U \frac{\partial X}{\partial \eta} \\
v & =U\left(\frac{v}{2 U x}\right)^{\frac{1}{2}}\left(\eta \frac{\partial X}{\partial \eta}-X+\zeta \frac{\partial X}{\partial \zeta}+2 \frac{\partial Z}{\partial \zeta}\right), \\
w & =-\frac{\delta^{*} U}{2 \pi}\left(\frac{v}{U x}\right)^{\frac{1}{2}}\left(\log \frac{U x}{v}\right) \frac{\partial Z}{\partial \eta} .
\end{aligned}
$$


Substituting (8) into (1) and taking the leading-order terms (which incidentally involves discarding inverse powers of $\log \frac{U x}{v}$ ) yields the following two key equations:

$$
\begin{aligned}
& \frac{\partial^{3} X}{\partial \eta^{3}}+\frac{\partial^{2} X}{\partial \eta^{2}}\left(X-\zeta \frac{\partial X}{\partial \zeta}-2 \frac{\partial Z}{\partial \zeta}\right)+\frac{\partial^{2} X}{\partial \eta \partial \zeta}\left(\zeta \frac{\partial X}{\partial \eta}+2 \frac{\partial Z}{\partial \eta}\right)=0 \\
& \frac{\partial^{3} Z}{\partial \eta^{3}}+\frac{\partial^{2} Z}{\partial \eta^{2}}\left(X-\zeta \frac{\partial X}{\partial \zeta}-2 \frac{\partial Z}{\partial \zeta}\right)+\frac{\partial^{2} Z}{\partial \eta \partial \zeta}\left(\zeta \frac{\partial X}{\partial \eta}+2 \frac{\partial Z}{\partial \eta}\right)=1-\frac{\partial X}{\partial \eta} \frac{\partial Z}{\partial \eta}
\end{aligned}
$$

The boundary conditions on $X$ and $Z$ are:

$$
Z=X=\frac{\partial X}{\partial \eta}=\frac{\partial Z}{\partial \eta}=0 \quad \text { on } \eta=0, \zeta>0
$$

corresponding to the no-slip and impermeability conditions, together with

$$
Z=X=\frac{\partial^{2} X}{\partial \eta^{2}}=\frac{\partial^{2} Z}{\partial \eta^{2}}=0 \text { on } \eta=0, \zeta<0,
$$

since the flow is symmetrical about $\eta=0$ off the plate. Note that the leading-order (dimensional) pressure term is $O\left(\frac{\rho v U}{x} \log ^{2}\left(\frac{U x}{v}\right)\right)$, and is independent of the transverse boundary-layer coordinate $\eta$.

It is clear that as $\eta \rightarrow \infty$, the freestream conditions must apply ( $u \rightarrow U$, and $w \rightarrow \frac{\partial \Phi}{\partial z}$ as given in (5b)), i.e. in this limit

$$
\frac{\partial X}{\partial \eta} \rightarrow 1, \quad \frac{\partial Z}{\partial \eta} \rightarrow 1
$$

Let us now consider the limit $\zeta \rightarrow \infty$ (i.e. close to the plate surface, distant from the side edge). It was shown in S61 that in this limit, the response is a Blasius flow (with a crossflow component)

$$
\frac{\partial X}{\partial \eta} \rightarrow \frac{\partial F}{\partial \eta}, \quad \frac{\partial Z}{\partial \eta} \rightarrow \frac{\partial L}{\partial \eta},
$$

where $F(\eta)$ is described by the Blasius equation, namely

$$
F^{\prime \prime \prime}+F F^{\prime \prime}=0, \quad F(0)=F^{\prime}(0)=0, \quad F^{\prime}(\infty)=1,
$$

whilst $L(\eta)$ is described by

$$
L^{\prime \prime \prime}+F L^{\prime \prime}+F^{\prime} L^{\prime}=1, \quad L(0)=L^{\prime}(0)=0, \quad L^{\prime}(\infty)=1 .
$$

In the far-field, in the off-plate direction, the work of S61 suggested that freestream conditions should be attained, namely (10). However, it is our assertion here (discussed below) that there are at least two solutions to the problem, only one of which fully attains freestream conditions in this limit.

\subsection{The features predicted by Stewartson}

The analysis of S61 introduced the quantity

$$
\kappa(\zeta, \eta)=-\left(\zeta \frac{\partial X}{\partial \eta}+2 \frac{\partial Z}{\partial \eta}\right),
$$

and noted that (9) depends crucially on the sign of $\kappa$. If $\kappa>0$ at some location $\zeta=\zeta^{*}$ for all values of $\eta$, then the parabolic nature of (9) is sufficient for us to conclude that the solution at $\zeta^{*}$ can only be influenced by the state of the system for $\zeta<\zeta^{*}$, that is, the propagation of disturbances is in the direction of increasing $\zeta$. Conversely, if $\kappa<0$ at some location $\zeta=\zeta^{*}$ for all values of $\eta$, then the solution at that point is only influenced by $\zeta>\zeta^{*}$, that is, the propagation of disturbances is in the direction of decreasing $\zeta$. If there are locations $\zeta=\zeta^{*}$ for which $\kappa$ changes sign at least once at some $\eta$ location, then the diffusion in $\eta$ present 
in (9) is sufficient to ensure that the solution at this location will depend on the state of the system in both directions.

In the far-field (on the plate), i.e. $\zeta \rightarrow \infty$

S61 did solve for $F(\eta)$ and $L(\eta)$, via (12) and (13), numerically. Clearly, $\kappa(\zeta>0, \eta=0)=0$, and the 'first' point of interest on decreasing $\zeta$ is therefore where $\kappa_{\eta}(\zeta, \eta=0)=0$, which occurs at $\zeta=\zeta_{0} \equiv$ $-2 L^{\prime \prime}(0) / F^{\prime \prime}(0)$. S61 gives a value of $\zeta_{0} \approx 5.02$; in fact, a more accurate value found herein is $\zeta_{0} \approx 5.18$. The implication in S61 was that $\kappa<0$ over some range of $\eta$ for $\zeta<\zeta_{0}$; however, we shall see in our work that this is not true. The results we present later indicate that the solution is rather more complex in the neighbourhood of $\zeta=\zeta_{0}$ than one would infer from S61.

In the far-field (off the plate), i.e. $\zeta \rightarrow-\infty$

S61 assumed that in the far-field off the plate (that is, as $\zeta \rightarrow-\infty$ ), the flow approaches the freestream values. However, our computational results (to be presented later) indicate that there are two possibilities. As $\zeta \rightarrow-\infty$, one can seek a more general solution of the form $X \sim \eta, Z \sim \tilde{Z}(\eta)$, where

$$
\tilde{Z}^{\prime \prime \prime}+\eta \tilde{Z}^{\prime \prime}+\tilde{Z}^{\prime}=1
$$

subject to $\tilde{Z}^{\prime} \rightarrow 1$ as $\eta \rightarrow \infty$, and $\tilde{Z}(0)=\tilde{Z}^{\prime \prime}(0)=0$. This admits a solution of the form

$$
\tilde{Z}^{\prime}(\eta)=1-\lambda e^{-\frac{1}{2} \eta^{2}}
$$

for any real constant $\lambda$, which must be determined as part of the global solution. Our numerical results indicate that there is a solution with $\lambda=0$ as suggested in S61; however, there is also an alternative state with $\lambda \neq 0$. As we shall see, the solution with $\lambda=0$ has a discontinuity in $\zeta$ derivatives at a critical location $\left(\zeta=\bar{\zeta}_{0}\right)$ off the plate, whilst the $\lambda \neq 0$ state does not. For the solution with $\lambda \neq 0$, it is only the leading-order velocity component $(u)$ that approaches its freestream value; as $\zeta \rightarrow-\infty$ there is a displacement effect on the crossflow $(w)$ velocity component connected to (16).

$$
\text { At the side edge, i.e. } \zeta=0
$$

Along the side edge of the plate, S61 discussed the three possibilities of $\kappa(\zeta=0, \eta)$ being positive for all $\eta$, negative for all $\eta$ or having a change in sign at a finite value of $\eta$. In S61 (page 9), it was suggested that the last of these three possibilities was the most likely. However, S61 notes that if $\kappa<0$, then $X_{\eta \eta}, Z_{\eta \eta}$ would be smooth for $\zeta>0$, whilst $X_{\eta}, Z_{\eta}$ would have singularities with exponent $\frac{1}{2}$. Our numerical results will show that there is no such sign change in $\kappa$ along $\zeta=0$. We suggest an asymptotic structure around $\zeta=0$ later in this paper.

\section{Numerical techniques and results}

We begin our numerical investigation with a somewhat simplistic homotopy approach. In (9), we will add the arbitrary terms $\epsilon \frac{\partial^{3} X}{\partial \zeta^{2} \partial \eta}$ and $\epsilon \frac{\partial^{3} Z}{\partial \zeta^{2} \partial \eta}$ to the $X$ and $Z$ equations, respectively, where $\epsilon$ is a constant. This approach has the significant advantage of making the problem fully elliptic throughout the entire domain; given that we expect the original parabolic system to be bidirectional, in at least some part of the flow domain, this does not seem like an unreasonable approach. As we shall show, this method proves to be surprisingly effective and provides a crucial starting point that will allow us to piece together the global solution structure via a combination of asymptotic methods and further numerical studies. However, as we shall see, there are a number of subtle features in the flow that will require significantly more effort to clarify.

Our aim is to perform the obvious homotopy continuation, reducing from $\epsilon=1$ towards $\epsilon=0$. Some caution is required with this approach as there is an implicit assumption that solutions achieved in the limit of $\epsilon \rightarrow 0$ remain solutions of the $\epsilon \equiv 0$ parabolic system. In order to address this issue, we will subsequently compare our numerical results with asymptotic descriptions of (9) in a number of key regions of the flow domain. Furthermore, we also use the $\epsilon \ll 1$ results as an initial guess to the original $(\epsilon \equiv 0)$ system (9); in 
regions where the parabolic system is unidirectional, it is straightforward to solve (9) via a standard CrankNicolson method (using one of the $\epsilon \ll 1$ solutions at a finite $\zeta$ location as a starting profile) as an independent check and interpretation of our results.

It is perhaps worth noting at this point that all local methods of solving this problem failed. In particular, attempts at sweeping through the computational domain (with $\epsilon=0$ ) using windward differencing of the $\zeta$ derivatives at fixed $\zeta$ locations proved to be entirely unsuccessful. The complexity of the flow structure and subtleties of the parabolic system necessitate a global solution approach, which solves for all variables at all points in the flow as a fully coupled system.

Our numerical scheme (for finite values of $\epsilon$ ) solves for the global flow field by applying second-order finite differencing over a uniformly distributed mesh of nodal points in the $\zeta-\eta$ plane. Typical computational parameters extend up to 4001 nodes in $\zeta$ and 121 nodes in $\eta$, over a domain $(\zeta, \eta) \in[-3,6] \times[0,6]$. We employ Newton iteration to solve the global system by a sparse matrix solver and the SuperLU library; all the results presented are independent of the computational mesh to the graphical accuracy of the figures. It is extremely challenging to solve this system owing to the large number of degrees of freedom, critical points in the flow and the discontinuity in the boundary conditions at the plate's edge (together with the inherent non-linearity).

\subsection{The $\lambda=0$ solution}

We begin with the off-plate conditions proposed in S61, namely freestream conditions with $\lambda=0$ in (16). As we have already discussed, of key importance to the global solution is the sign of $\kappa$ through the two-dimensional flow domain. The sign of $\kappa$ appears to change first in the neighbourhood of $\eta=0$; hence, in Fig. 2 we show the distributions of $\kappa(\zeta<0, \eta=0)$ and $\kappa_{\eta}(\zeta>0, \eta=0)$ over a range of values of the homotopy parameter $\epsilon \leq 1.6 \times 10^{-3}$.

From Fig. 2, at the values of $\epsilon$ shown, the bulk of the solution between $\zeta=0$ (the side edge of the plate) and $\zeta=\zeta_{1} \approx 4.8$ is largely independent of $\epsilon$. There is still some variation off the plate $(\zeta<0)$ at these values of $\epsilon$; typically smaller values of $\epsilon$ are required before such variations are reduced. We also note that there are some much smaller-scale variations in the region $\zeta_{1} \lesssim \zeta \lesssim \zeta_{0}$ (as shown by the enlarged view presented in Fig. 2b).

Of particular interest is the behaviour near $\zeta=\zeta_{0}$, where $\zeta_{0}=-2 L^{\prime \prime}(0) / F^{\prime \prime}(0) \approx 5.18$. As $\epsilon$ is decreased we observe that, counter to the assumptions made in $\mathrm{S} 61, \kappa_{\eta}(\zeta, \eta=0)$ does not change sign at $\zeta_{0}$, rather it appears to approach zero, but then immediately recovers, only changing sign at a secondary location (denoted in Fig. 2(b) by $\zeta=\zeta_{1} \approx 4.8$ ) that is slightly closer to the side edge of the plate.

The same qualitative behaviour is observed off the plate, with the distribution of $-\kappa(\zeta, \eta=0)$ showing a marked minimum near zero (at $\zeta \approx-0.95$ ), before increasing again, and eventually changing sign at a secondary location $\zeta=-2$.

In Fig. 3, we show the all-important contours that delineate the $\zeta-\eta$ flow domain into regions of $\kappa<0$ and $\kappa>0$. When $\epsilon=0$, there is only diffusion in the $\eta$ coordinate and, as discussed previously, $\kappa>0$ leads to the propagation of disturbances to the right, whilst $\kappa<0$ leads to propagation of disturbances to the left. The far-field conditions on the 'right' $(\zeta \gg 1)$ and on the 'left' $(-\zeta \gg 1)$ both influence the global solution owing to the signs of $\kappa$ in those regions. On the plate $(\zeta>0)$, there is a single region in which $\kappa$ is positive. This 'reverse' region exists between the side edge at $\zeta=0$ and a critical location $\zeta=\zeta_{1}$. These results are not in line with the discussion of S61, as they indicate that (i) there is no zero of $\kappa$ at a finite $\eta$ at the side-edge $\zeta=0$ and (ii) the $\kappa>0$ region does not extend to $\zeta=\zeta_{0} \approx 5.18$, rather it terminates at $\zeta=\zeta_{1} \approx 4.8$.

From Figs. 2 and 3, we have some confidence in the global solution and the direction of the propagation of disturbances in the parabolic system. However, it is apparent that, local to some critical spanwise positions, the solution is more sensitive to the homotopy parameter than at other positions (this is perhaps not unexpected). This local variation is highlighting subtleties associated with the underlying parabolic system, as we will demonstrate below at several critical $\zeta$ locations, beginning at $\zeta=\zeta_{0}$.

\subsubsection{Short-scale eigenmodes near $\zeta=\zeta_{0}$}

For sufficiently large values of $\zeta$, the solution for $X$ and $Z$ is $\zeta$-independent and simply a Blasius streamwise profile and an associated crossflow induced by the finite span of the plate:

$$
X(\zeta, \eta)=F(\eta), \quad Z(\zeta, \eta)=L(\eta), \quad \text { for } \zeta>\zeta_{0} ;
$$



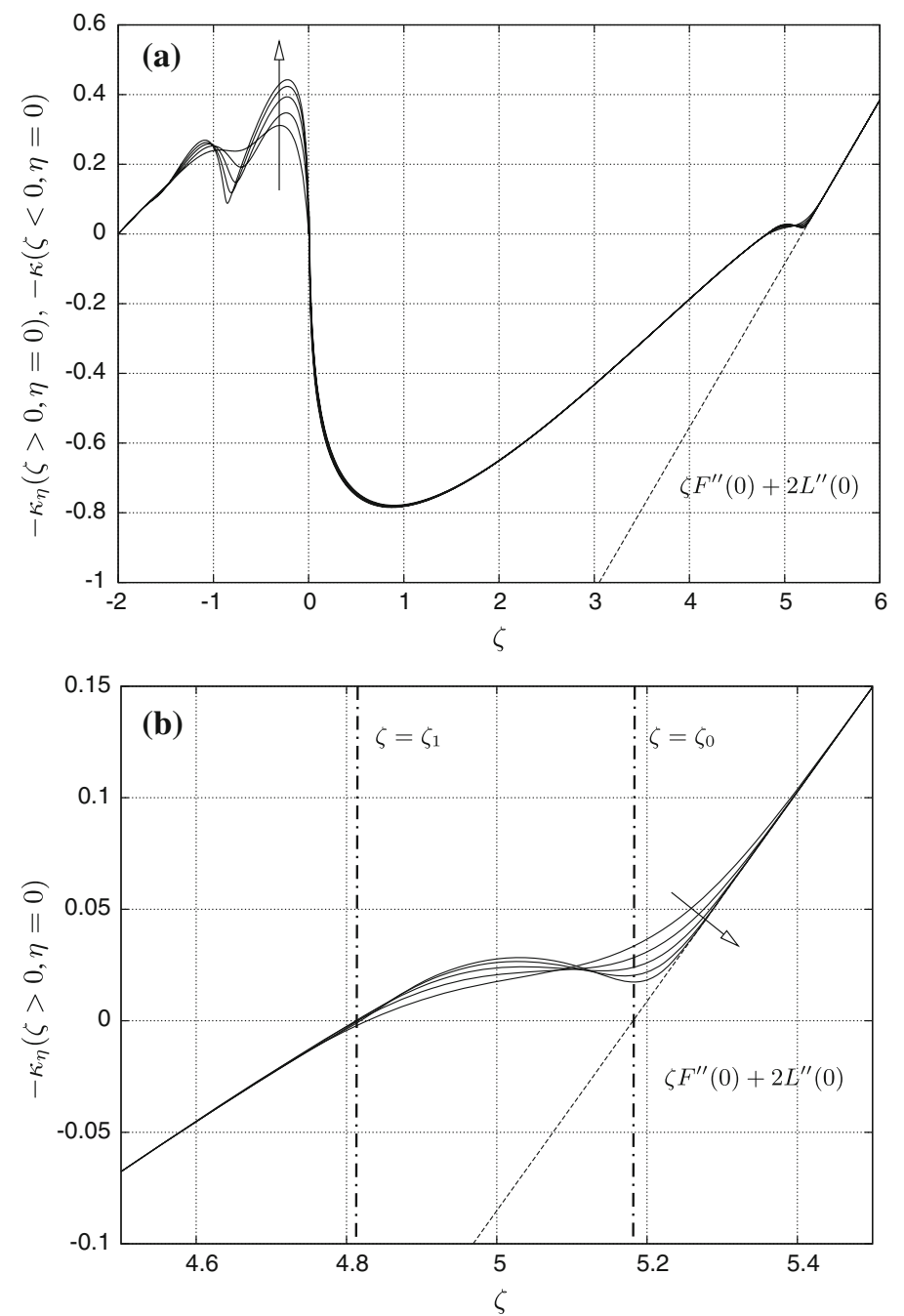

Fig. 2 a The on-plate distribution of $-\kappa_{\eta}(\zeta>0, \eta=0)$ and the off-plate distribution of $-\kappa(\zeta<0, \eta=0)$. A change in sign of either quantity indicates a reversal in the direction of disturbance propagation in the parabolic system (9b). The solutions are obtained with finite $\epsilon=1.6 \times 10^{-3}, 8 \times 10^{-4}, 4 \times 10^{-4}, 2 \times 10^{-4}, 1 \times 10^{-4}$; the arrow indicates decreasing $\epsilon$. Also shown (as the dotted line) is the predicted far-field behaviour on the plate $-\kappa_{\eta}=\zeta F^{\prime \prime}(0)+2 L^{\prime \prime}(0)$. b The on-plate distribution in a small region near $\zeta=\zeta_{0}$

here $F$ and $L$ are easily determined from (12) and (13). Therefore, on the plate when $\epsilon=0$

$$
\kappa_{\eta}\left(\zeta>\zeta_{0}, \eta=0\right)=\zeta F^{\prime \prime}(0)+2 L^{\prime \prime}(0)
$$

which has a zero when $\zeta$ is

$$
\zeta_{0} \equiv-2 L^{\prime \prime}(0) / F^{\prime \prime}(0) \approx 5.18
$$

In S61, the assumption was that $\zeta=\zeta_{0}$ is associated with the beginning of a $\kappa>0$ region near the plate surface; however, the solution structure is somewhat more complicated.

The solution (17) solves (9) for all $\zeta>0$; however, it only plays a role in the global solution for $\zeta>\zeta_{0}$, as is clearly demonstrated in the results of Fig. 2. The obvious question is how this simple solution connects to the global solution as $\epsilon \rightarrow 0$. As noted in S61, there are some striking similarities with the flow adjacent to an impulsively started semi-infinite flat plate; see $[11,14]$ and also [8]. When $\epsilon \equiv 0$ in the problem herein, for $\zeta>\zeta_{0}$ there is a simple $\zeta$-independent solution, whilst for $\zeta<\zeta_{0}$ there is a response that should clearly be $\zeta$ dependent. In the case of an impulsively started semi-infinite flat plate [11], a similar variable $(\tau)$ exists, which describes the transition between a $\tau$-dependent Blasius flow (near the leading edge) and a $\tau$-independent 


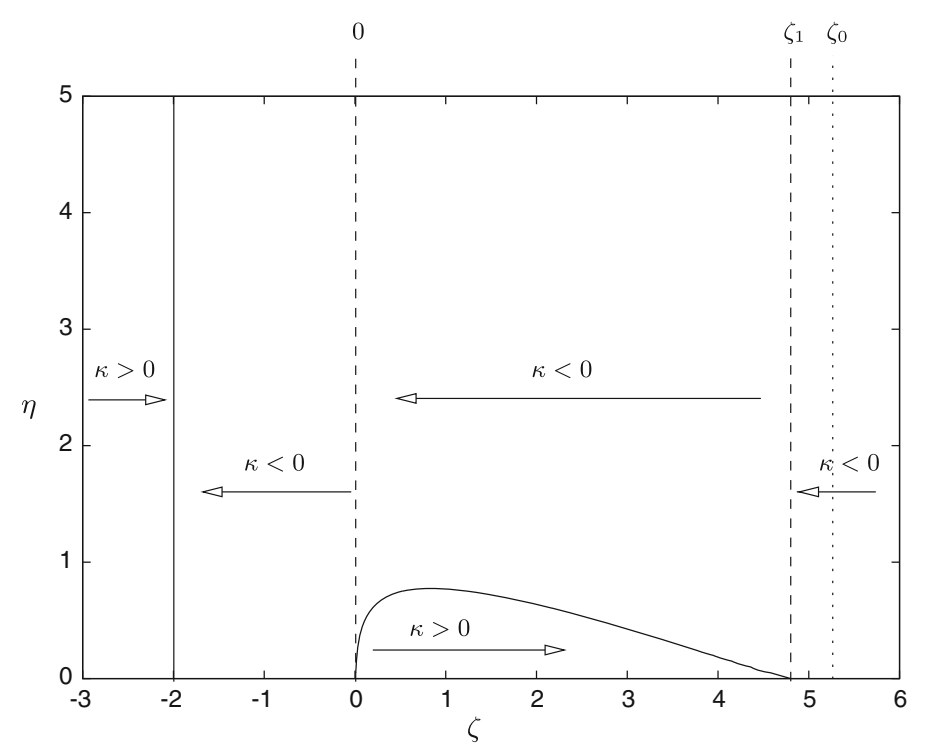

Fig. 3 The delineation of the $\zeta-\eta$ flow domain into regions of $\kappa>0$ and $\kappa<0$ for the $\lambda=0$ state. Note that the plate exists along $\eta=0, \zeta>0$. In regions of $\kappa<0$, disturbances propagate to the left, whilst for $\kappa>0$, disturbances propagate to the right. The figure shows the boundaries for the $\lambda=0$ solution

Rayleigh layer (sufficiently far downstream). The transition cannot be described in terms of a series expansion, leaving either a discontinuity in derivatives or an essential singularity as the mechanism of reconciling the two regions at some critical point. It is the presence of an essential singularity that is speculatively suggested to exist both in S61 and in [11]. To shed some light on this issue for the present problem, it is instructive to formulate a linearised perturbation problem.

The far-field solution (17) can, in principle, be parabolically marched towards the side edge of the plate (in the direction of decreasing $\zeta$ ) and suggests a linearised perturbation analysis of the form:

$$
\begin{aligned}
X(\zeta, \eta) & =F(\eta)+\delta f(\eta) E(\zeta), \\
Z(\zeta, \eta) & =L(\eta)+\delta l(\eta) E(\zeta),
\end{aligned}
$$

where $\delta \ll 1$ and

$$
E(\zeta)=\exp \left\{\int_{\zeta}^{\infty} \sigma(\bar{\zeta}) \mathrm{d} \bar{\zeta}\right\}
$$

This results in a local eigenproblem for the growth rate $\sigma$, parameterised by the location $\zeta$, governed by

$$
\begin{aligned}
& f^{\prime \prime \prime}+\sigma\left[(\zeta f+2 l) F^{\prime \prime}-\left(\zeta F^{\prime}+2 L^{\prime}\right) f^{\prime}\right]=-f F^{\prime \prime}-f^{\prime \prime} F, \\
& l^{\prime \prime \prime}+\sigma\left[(\zeta f+2 l) L^{\prime \prime}-\left(\zeta F^{\prime}+2 L^{\prime}\right) l^{\prime}\right]=-l^{\prime \prime} F-L^{\prime \prime} f-F^{\prime} l^{\prime}-f^{\prime} L^{\prime} .
\end{aligned}
$$

This 'parallel flow' analysis is obviously ad hoc in general but becomes rigorous for increasingly short-scale modes where $|\sigma| \gg 1$, which are the topic of interest here.

Solution of (21) for $\zeta>\zeta_{0}$ shows that there are two eigenvalues with positive real part for $5.21 \lesssim \zeta \lesssim 5.25$ (a complex conjugate pair). The conjugate pair become real for $\zeta_{0}<\zeta \lesssim 5.21$, with the smaller value remaining $O(1)$ whilst the larger value tends to infinity as $\zeta \rightarrow \zeta_{0}$, as shown in Fig. 4. For $\zeta<\zeta_{0}$ only the lower eigenvalue persists and is joined by (infinitely) many other eigenvalues that arise 'from infinity'; because the underlying solution (17) is irrelevant in terms of the global solution for $\zeta<\zeta_{0}$, we do not present these results.

An asymptotic description of this short-scale eigenmode is presented later in Sect. 4.1, in the limit of $\sigma \rightarrow \infty$. However, for the present, we simply note that, for $\zeta=\zeta_{0}+\Delta \zeta$, with $0<\Delta \zeta \ll 1$, a linearised perturbation to the baseflow solution $\{F, L\}$ develops in the form

$$
\exp \left\{\frac{3 \sigma_{0}}{\left(\zeta-\zeta_{0}\right)^{\frac{1}{3}}}\right\}
$$




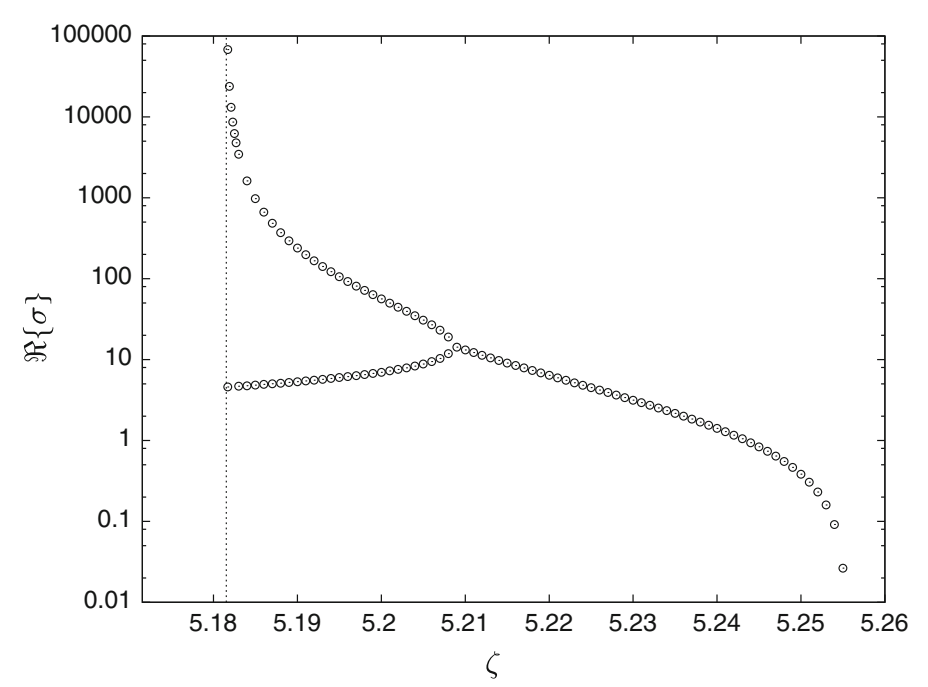

Fig. 4 The development of $\Re\{\sigma\}$ as $\zeta \rightarrow \zeta_{0}^{+}$, showing the singular (real) eigenvalue at the critical point $\zeta_{0}$; the value of $\zeta_{0}$ is shown by the vertical line

for some constant $\sigma_{0}$. This short-scale eigenmode provides a mechanism for disruption of the $\zeta$-independent solution $X=F, Z=L$, beyond the critical point $\zeta_{0}$ into the region $\zeta<\zeta_{0}$.

\subsubsection{Parabolic marching for $\zeta_{1}<\zeta<\zeta_{0}$}

The above discussion of the short-scale mode that can be found at $\zeta=\zeta_{0}$ suggests that we examine the following perturbed parabolic problem. We solve for $X(\zeta, \eta)$ and $Z(\zeta, \eta)$ by starting the computation at some chosen $\zeta=\zeta_{i}>\zeta_{0}$ with an initial solution

$$
\begin{aligned}
X\left(\zeta_{i}, \eta\right) & =F(\eta)+\delta f(\eta), \\
Z\left(\zeta_{i}, \eta\right) & =L(\eta)+\delta l(\eta),
\end{aligned}
$$

where $f$ and $l$ correspond to the short-scale eigenmode of (21), as evaluated at $\zeta=\zeta_{i}$. We choose to normalise the eigenmode such that $f(\eta \rightarrow \infty) \rightarrow 1$.

Using these initial conditions, we then march (using a Crank-Nicolson scheme) in the direction of decreasing $\zeta$. Clearly, if $\delta \equiv 0$, then the computation should terminate at $\zeta=\zeta_{0}$, at which point S61 assumed $\kappa$ would change sign local to the boundary $\eta=0$, with the solution for $\zeta<\zeta_{0}$ only being determined by a global solution method. However, the short-scale eigenmodes present at $\zeta=\zeta_{0}$ lead to an alternative solution being realised, which can in fact be fully determined by parabolic marching, at least until a secondary location $\zeta=\zeta_{1}<\zeta_{0}$ is encountered.

The numerical solution for $\kappa_{\eta}$ evaluated at the plate is shown in Fig. 5 for a computation started at $\zeta=\zeta_{i}=5.2$ and $\delta=10^{-8}$; the exact choice of $\zeta_{i}$ has very little influence for $\delta$ sufficiently small. Very small steps in $\zeta\left(\approx 10^{-4}\right)$ are required in the Crank-Nicolson computations to resolve the very rapid growth near $\zeta=\zeta_{0}$. In the same figure, we also show the finite- $\epsilon$ numerical results obtained from the global solver.

In practice, a sensible approach is to compute using the Crank-Nicolson scheme described above wherever it is possible to do so and then impose the resulting profile as a Dirichlet condition in the global solver at $\zeta=\zeta_{1}$ (where $\zeta_{1}$ is determined as part of the parabolic scheme).

As $\zeta \rightarrow \zeta_{1}$ the $\epsilon \equiv 0$ solution determined by the Crank-Nicolson scheme again suggests a singular $\zeta$ derivative at the point where $\kappa_{\eta}\left(\zeta=\zeta_{1}, \eta=0\right)=0$, as can be observed in Fig. 5. This feature is associated with a rapid but very small $\left(\approx 10^{-2}\right)$ change in the second derivative of $X$ and $Z$ at the plate and is not observed in the global solution even for $\epsilon=10^{-4}$. Some care in the interpretation of the structure of the system is required at $\zeta=\zeta_{1}$, and we will return to this region for further discussion in Sect. 4.3 below. 


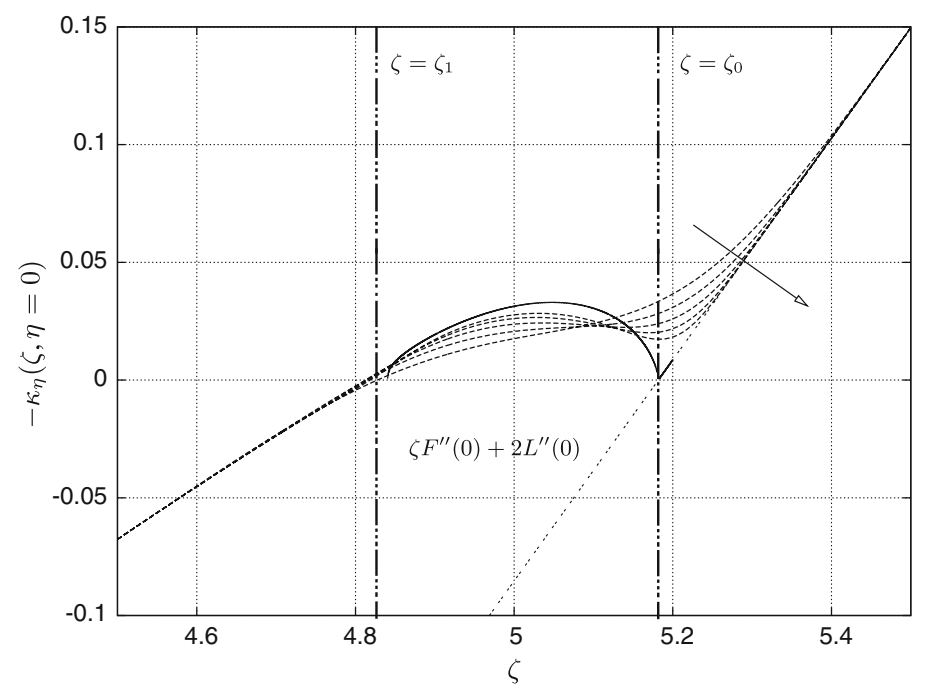

Fig. 5 The solid line represents the $\epsilon \equiv 0$ Crank-Nicolson results, whilst the dashed lines are the global solver results for $\epsilon=1.6 \times 10^{-3}, 8 \times 10^{-4}, 4 \times 10^{-4}, 2 \times 10^{-4}, 1 \times 10^{-4} ; \epsilon$ decreases in the direction of the arrow shown. The dotted line is the solution predicted for $\zeta>\zeta_{0}$

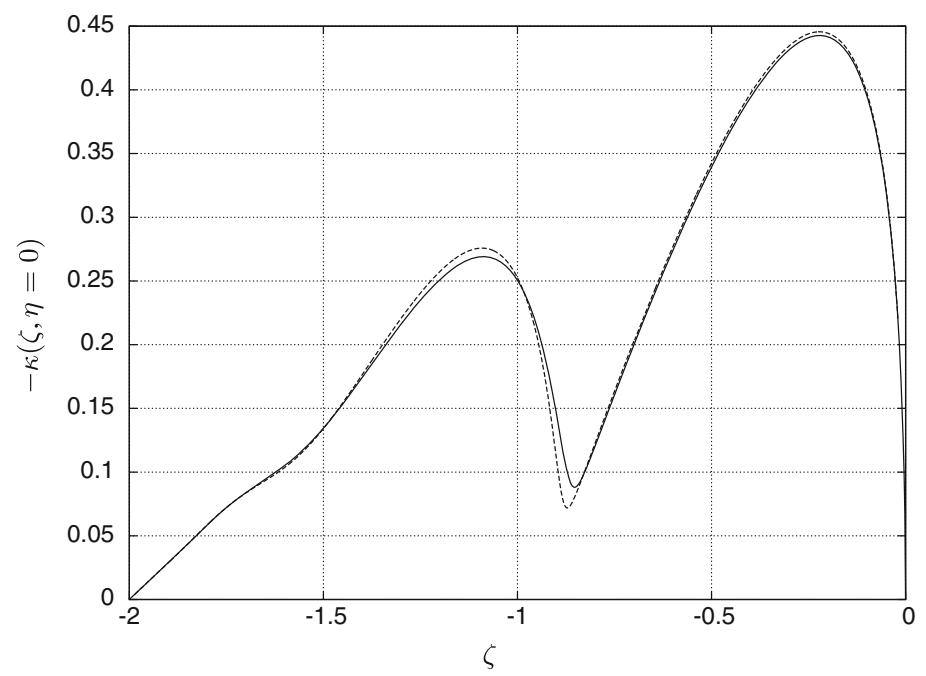

Fig. 6 A comparison between the $\epsilon=10^{-4}$ global solution (solid) and the corresponding $\epsilon \equiv 0$ data (dashed) obtained from parabolic marching for $-2<\zeta \leq \bar{\zeta}_{i}=2 \times 10^{-2}$

\subsubsection{Parabolic marching off the plate, $\zeta<0$}

Immediately off the side edge of the plate, the direction of propagation of disturbances is entirely in the direction of decreasing $\zeta$, as shown in Fig. 3. Hence, if we take a cross section of the global solution at some point $\zeta=\bar{\zeta}_{i}$ (where $\bar{\zeta}_{i}$ is small and negative), we can then again perform a parabolic marching procedure in the direction away from the plate as a check on the consistency of the homotopy approach.

The results of Fig. 6 clearly show that the $\epsilon=10^{-4}$ global solution and this parabolic marching process (with $\epsilon=0$ ) are in agreement. The rapid variation with $\zeta$ beyond $\zeta \approx-0.8$ is a property of the parabolic system and is clearly replicated in the $\epsilon=0$ results.

The behaviour observed off the plate is analogous (qualitatively) to that already described in the neighbourhood of $\zeta=\zeta_{0}^{+}$. Computation of local eigenmodes in the off-plate region similarly demonstrates that a short-scale eigenmode is found as $\kappa$ becomes smaller. This short-scale mode is again indicative of a singular $\zeta$ gradient and extreme sensitivity to upstream conditions. 


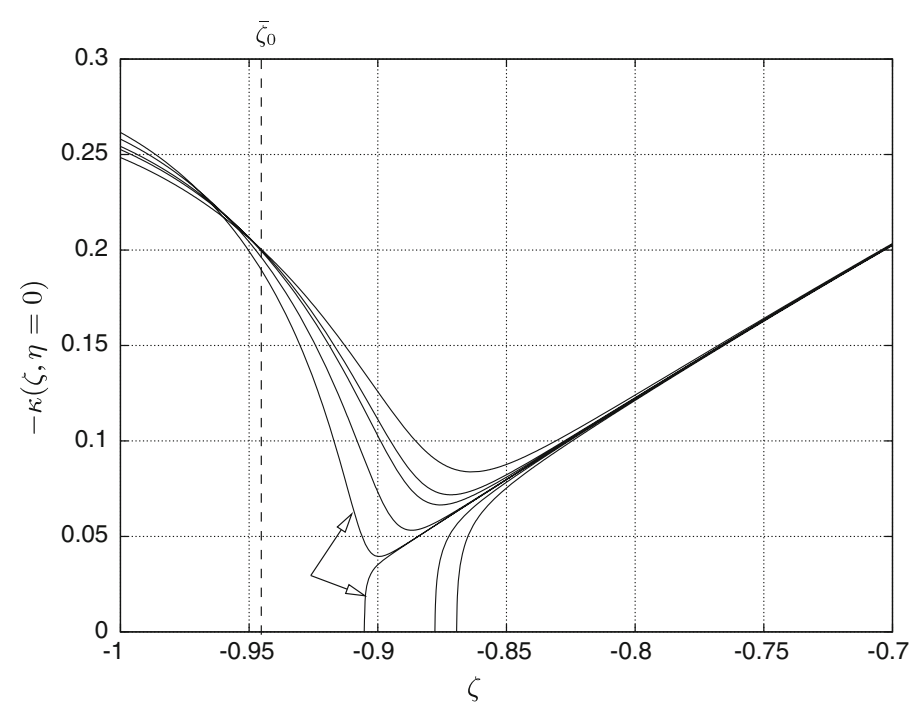

Fig. 7 The influence of small eigenmode perturbations to the $\epsilon=0$ solution during parabolic marching (right to left) in the off-plate region. The perturbation is applied at $\zeta^{*}=-0.7$. For the two distributions indicated by the arrows, the difference in the two values of $\kappa\left(\zeta^{*}, \eta=0\right)$ is $2.22 \times 10^{-7}$, clearly demonstrating a sensitivity to the upstream solution in this region

It is relatively straightforward to describe the singularity at $\zeta=\zeta_{0}$ because we know precisely what the underlying base flow solution is, via the ordinary differential equations for $F$ and $L$. However, in this off-plate region, the situation is rather more complicated because we can only determine the underlying solution globally, depending as it does on the bidirectional nature of the flow. Nevertheless, we can demonstrate a sensitivity of the solution in this off-plate region in a manner analogous to that of Sect. 3.1.2.

We propose that a global solution exists with $\kappa\left(\zeta=\bar{\zeta}_{0}, \eta=0\right)=0$, where $\bar{\zeta}_{0}$ is a critical point away from the plate $\left(\bar{\zeta}_{0} \approx-0.95\right)$. In Fig. 7 , we demonstrate the sensitive nature of the parabolic system in the neighbourhood of $\zeta=\bar{\zeta}_{0}$. To obtain this figure, we take a cross section of a finite $\epsilon$ solution $\left(X^{*}, Z^{*}\right)$ at some $\zeta=\zeta^{*}(=-0.7)$ and compute the corresponding largest local eigenvalue $\sigma^{*}$ and eigenmode $\left(f^{*}, l^{*}\right)$. We then march the parabolic system (9), in the direction of decreasing $\zeta$, starting from the initial conditions

$$
\left(X\left(\zeta^{*}, \eta\right), Z\left(\zeta^{*}, \eta\right)\right)=\left(X^{*}(\eta), Z^{*}(\eta)\right)+\delta\left(f^{*}(\eta), l^{*}(\eta)\right)
$$

for a range of amplitudes $\delta$, with the eigenfunction normalised such that $l^{*}(\infty)=1$. A suitable addition of this eigenmode, which ultimately feeds into an asymptotically short-scale mode of the linearised problem at $\bar{\zeta}_{0}$, allows the parabolic marching to proceed ever closer to the singular point $\zeta=\bar{\zeta}_{0}$ where $\kappa=0$. Two distinct behaviours emerge near $\bar{\zeta}_{0}$; in one, $\kappa(\eta=0)$ reaches a minimum value, close to zero, but then sharply recovers (the behaviour observed in Fig. 6), whereas in the other, $\kappa(\eta=0)$ approaches zero. This behaviour is associated with a singularity of the parabolic system where $\kappa(\eta=0)=0$, at which point eigenmodes exist that can be associated with non-uniqueness of the global solution, as we shall discuss below.

\subsection{The $\lambda \neq 0$ solution}

The description of the previous section suggests that non-uniqueness may be present in the solutions of (9). By imposing $\lambda=0$, we have thus far constructed a global solution, but this solution appears to include a discontinuity of $\zeta$ derivatives at the point $\zeta=\bar{\zeta}_{0}$. This discontinuity, although consistent with the $\lambda=0$ freestream conditions favoured by S61, is not a necessary feature if we allow $\lambda \neq 0$.

We first consider the influence of varying $\lambda$. At general values of $\lambda>0$, a flow field can be computed at finite values of $\epsilon$, but typically these do not yield a continuous solution in the limit of $\epsilon \rightarrow 0$. An illustration of this is given in Fig. 8, which shows the variation of $Z_{\eta}(\zeta, \eta=0)$, for the particular (arbitrary) choice $\lambda=0.2$. As can be observed, as $\epsilon$ decreases a region develops off the plate where the ad hoc diffusive terms (as introduced for the homotopy approach) remain dominant for all $\epsilon$. We speculate that, for general values of $\lambda$, solutions of the $\epsilon=0$ system would necessarily possess a discontinuity in $X$ and $Z$ (rather than just in the 


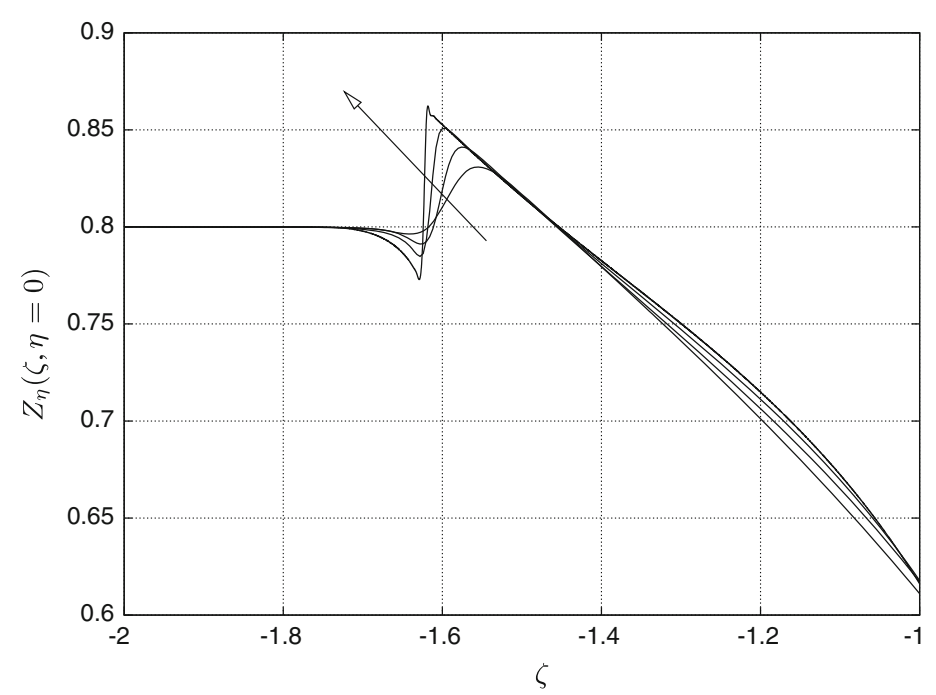

Fig. 8 An example of how arbitrary values of $\lambda$ do not result in continuous solutions to the parabolic problem as $\epsilon \rightarrow 0$. Here, we show $Z_{\eta}(\zeta<0, \eta=0)$ for an ad hoc choice of $\lambda=0.2$. The data are obtained with $\epsilon=0.0016,0.0008,0.0004,0.0002$, decreasing in the direction of the arrow shown

$\zeta$ derivatives as found in the $\lambda=0$ case). However, there is a critical value of $\lambda \approx 0.51$ for which a solution with a smooth variation of $X$ and $Z$ can be obtained.

In Fig. 9 we show both the $\lambda \approx 0.51$ and the $\lambda=0$ solutions, which combine at $\zeta=\bar{\zeta}_{0}$; both solutions are identical in the region $\zeta>\bar{\zeta}_{0}$. In the same figure, we also show a $\epsilon=10^{-4}, \lambda=0$, global solution for reference; as was found to also occur near $\zeta_{0}$ (i.e. on the plate, Fig. 5), the finite $\epsilon$ obviously acts to smooth the discontinuity in $\zeta$ derivatives at the critical point $\bar{\zeta}_{0}$.

The $\lambda \neq 0$ solution has benign behaviour near $\bar{\zeta}_{0}$. The non-uniqueness that arises from the critical point $\zeta=\bar{\zeta}_{0}$ closely mirrors that obtained near $\zeta=\zeta_{0}$ (on the plate). The difference here is that both solutions can play a role in the global solution, whereas the solution (17) (in $\zeta<\zeta_{0}$ ) does not play a role in the global flow (noting that this solution cannot satisfy the edge condition at $\zeta=0$ ); this is a theme taken up in Sect. 4.6.

For $\lambda \neq 0$, the contours of $\kappa=0$ in the $\zeta-\eta$ domain are more complicated. In Fig. 10, we repeat Fig. 3, but for the $\lambda \approx 0.51$ state, showing that the previous contour at $\zeta=-2$ is now a curved boundary that must be determined numerically, and indeed globally.

\section{Asymptotic results}

In this section, we investigate in detail the behaviour of the flow in the vicinity of the critical locations suggested above, namely close to $\zeta=\zeta_{0}, \zeta=\zeta_{1}, \zeta=0, \zeta=\bar{\zeta}_{0}$ and $\zeta=-2$. The purpose of these analyses is to confirm the features observed in the numerical results. In the asymptotic descriptions below, it is convenient to introduce a new quantity

$$
G(\zeta, \eta)=\zeta X+2 Z
$$

where (9a) may be written

$$
X_{\eta \eta \eta}+X_{\eta \eta}\left(2 X-G_{\zeta}\right)+X_{\eta \zeta} G_{\eta}=0,
$$

whilst a combination of (9a) and (9b) yields

$$
G_{\eta \eta \eta}-G_{\eta \eta}\left(G_{\zeta}-2 X\right)+G_{\eta} G_{\eta \zeta}-\zeta X_{\eta}^{2}=2
$$

with $G(\eta=0, \forall \zeta)=G_{\eta}(\eta=0, \zeta>0)=G_{\eta \eta}(\eta=0, \zeta<0)=0$ and $G_{\eta} \rightarrow \zeta+2$ as $\eta \rightarrow \infty$. Note that in terms of this new variable, $\kappa \equiv-G_{\eta}$.

We now consider each of the critical $\zeta$ locations in turn, in the direction of $\zeta$ decreasing. 

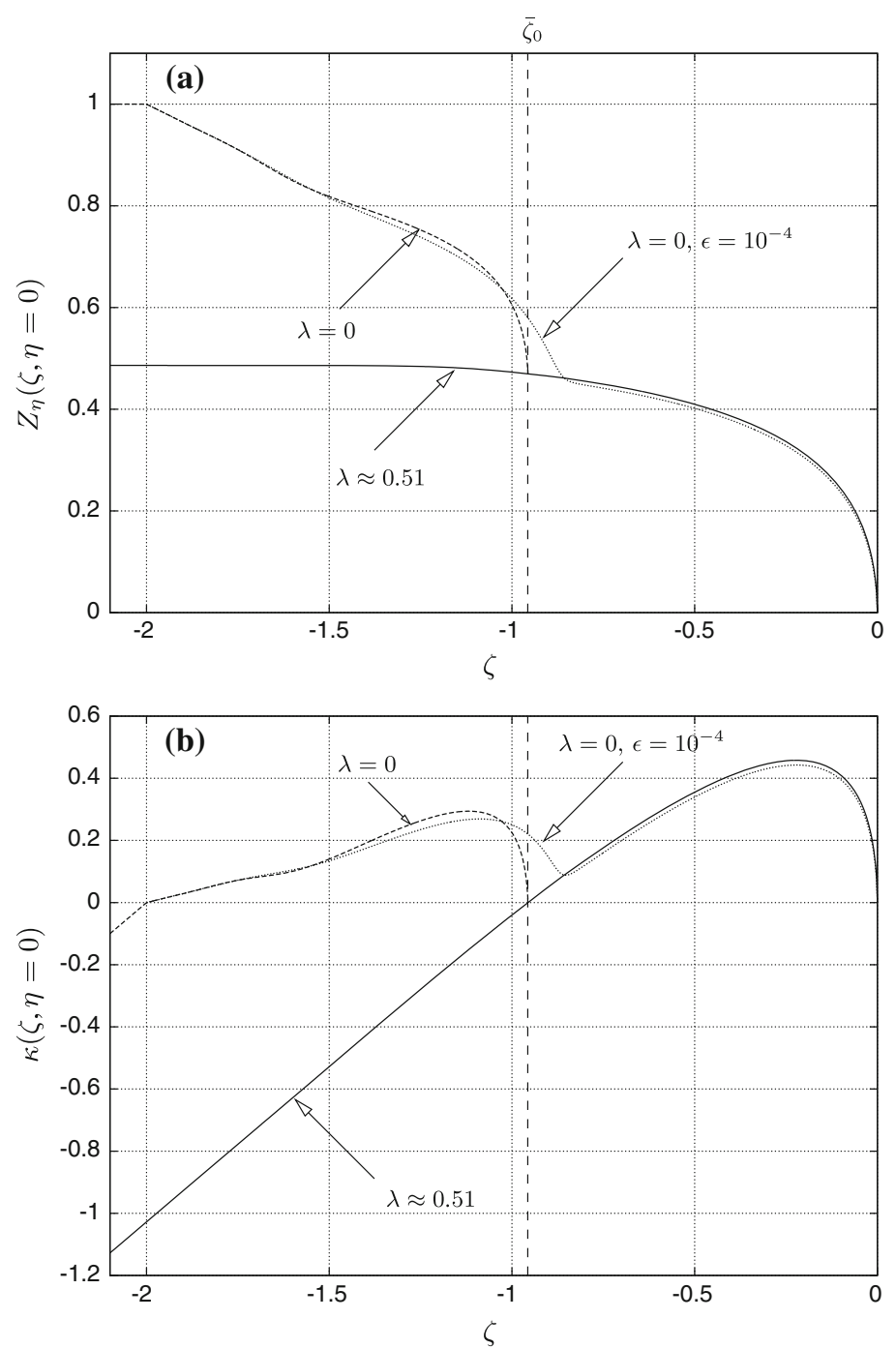

Fig. 9 The solution is non-unique in the region $\zeta<\bar{\zeta}_{0}$, with the two alternative states connecting to the same solution in the domain $\zeta>\bar{\zeta}_{0}$. The non-uniqueness arises at the critical point $\bar{\zeta}_{0} \approx-0.95$ where $\kappa\left(\zeta=\bar{\zeta}_{0}, \eta=0\right)=0$

4.1 The asymptotics of the short-scale eigenmodes at $\zeta=\zeta_{0}^{+}$

For $\zeta=\zeta_{0}+\Delta \zeta$, with $0<\Delta \zeta \ll 1$, as discussed earlier and illustrated in Fig. 4, we find a local eigenvalue $|\sigma(\zeta)| \gg 1$. The outer solution to the linear eigenproblem is straightforward

$$
f=F^{\prime}(\eta), \quad l=L^{\prime}(\eta),
$$

which clearly does not satisfy the plate conditions $f^{\prime}(\eta=0)=0$ and $l^{\prime}(\eta=0)=0$. There is therefore an inner layer, which asymptotic balancing predicts to be given by

$$
\eta=Y \sigma^{-\frac{1}{4}},
$$

where $Y=O(1)$, and

$$
\begin{aligned}
& F=F^{\prime \prime}(0) \frac{Y^{2}}{2} \sigma^{-\frac{1}{2}}-F^{\prime \prime}(0)^{2} \frac{Y^{5}}{5 !} \sigma^{-\frac{5}{4}}+\cdots, \\
& G=\frac{Y^{3}}{3} \sigma^{-\frac{3}{4}}+F^{\prime \prime}(0) Y^{2}\left\{\frac{1}{2} \Delta \zeta \sigma^{-\frac{1}{2}}-\zeta_{0} F^{\prime \prime}(0) \frac{Y^{3}}{5 !} \sigma^{-\frac{5}{4}}\right\}+\cdots,
\end{aligned}
$$




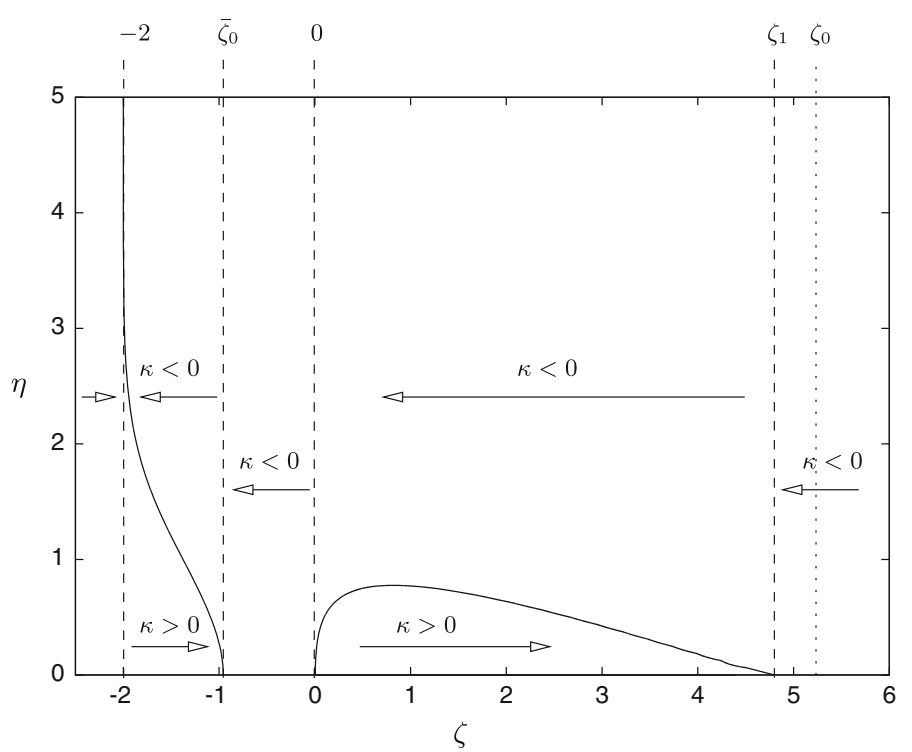

Fig. 10 The delineation of the $\zeta-\eta$ flow domain into regions of $\kappa>0$ and $\kappa<0$ for the $\lambda \neq 0$ solution. Note that the plate exists along $\eta=0, \zeta>0$. In regions of $\kappa<0$, disturbances propagate to the left, whilst for $\kappa>0$, disturbances propagate to the right

$$
\begin{aligned}
& f=f_{0}(Y) \sigma^{-\frac{1}{4}}+f_{1}(Y) \sigma^{-\frac{3}{4}}+\cdots, \\
& g=Y^{2} \sigma^{-\frac{1}{2}}+g_{1}(Y) \sigma^{-1}+\cdots .
\end{aligned}
$$

Here, $G=\zeta F+2 L$ as defined above, and $g=\zeta f+2 l$ is the corresponding perturbation quantity. We then find that the leading-order term for the perturbation in the inner layer satisfies

$$
f_{0}^{\prime \prime \prime}-Y^{2} f_{0}^{\prime}=-Y^{2} F^{\prime \prime}(0),
$$

subject to $f_{0}(0)=f_{0}{ }^{\prime}(0)=0$ and $f_{0}{ }^{\prime}(\infty)=F^{\prime \prime}(0)$. Although this provides the inner layer solution, it only requires that the eigenvalue is large and does not determine the functional relationship $\sigma=\sigma(\zeta)$. Nevertheless, solution of (30) shows good agreement with the rescaled numerical solution of (21) as $\sigma \rightarrow \infty$; a comparison is shown in Fig. 11.

The leading-order form for the eigenvalue must be obtained at higher order, although we do not pursue the problem further here. It is sufficient to note that the $O(\Delta \zeta)$ term is balanced at next order if we assume

$$
\sigma=\sigma_{0} \Delta \zeta^{-\frac{4}{3}}+\cdots,
$$

where $\sigma_{0}$ is a constant to be determined at higher order.

\subsection{The critical point $\zeta=\zeta_{0}^{-}$}

At this point $\kappa_{\eta}\left(\zeta=\zeta_{0}, \eta=0\right)=0$, which implies $G_{\eta \eta}\left(\zeta_{0}, 0\right)=\zeta_{0} F^{\prime \prime}(0)+2 L^{\prime \prime}(0)=0$. The outer flow in this region remains (at leading order) the far-field Blasius (with crossflow) together with an $O\left(\left(\zeta_{0}-\zeta\right)^{\frac{3}{4}}\right)$ perturbation:

$$
\begin{aligned}
& X=F(\eta)+\left(\zeta_{0}-\zeta\right)^{\frac{3}{4}} A_{0} F^{\prime}(\eta)+\cdots, \\
& G=\left[\zeta_{0} F(\eta)+2 L(\eta)\right]+\left(\zeta_{0}-\zeta\right)^{\frac{3}{4}} A_{0}\left[\zeta_{0} F^{\prime}(\eta)+2 L^{\prime}(\eta)\right]+\cdots .
\end{aligned}
$$

Here, the amplitude $A_{0}$ is a constant that will be determined at higher order when the non-linear influence of the perturbation is felt. We have deliberately chosen the $3 / 4$ power above to balance non-linearity and the 

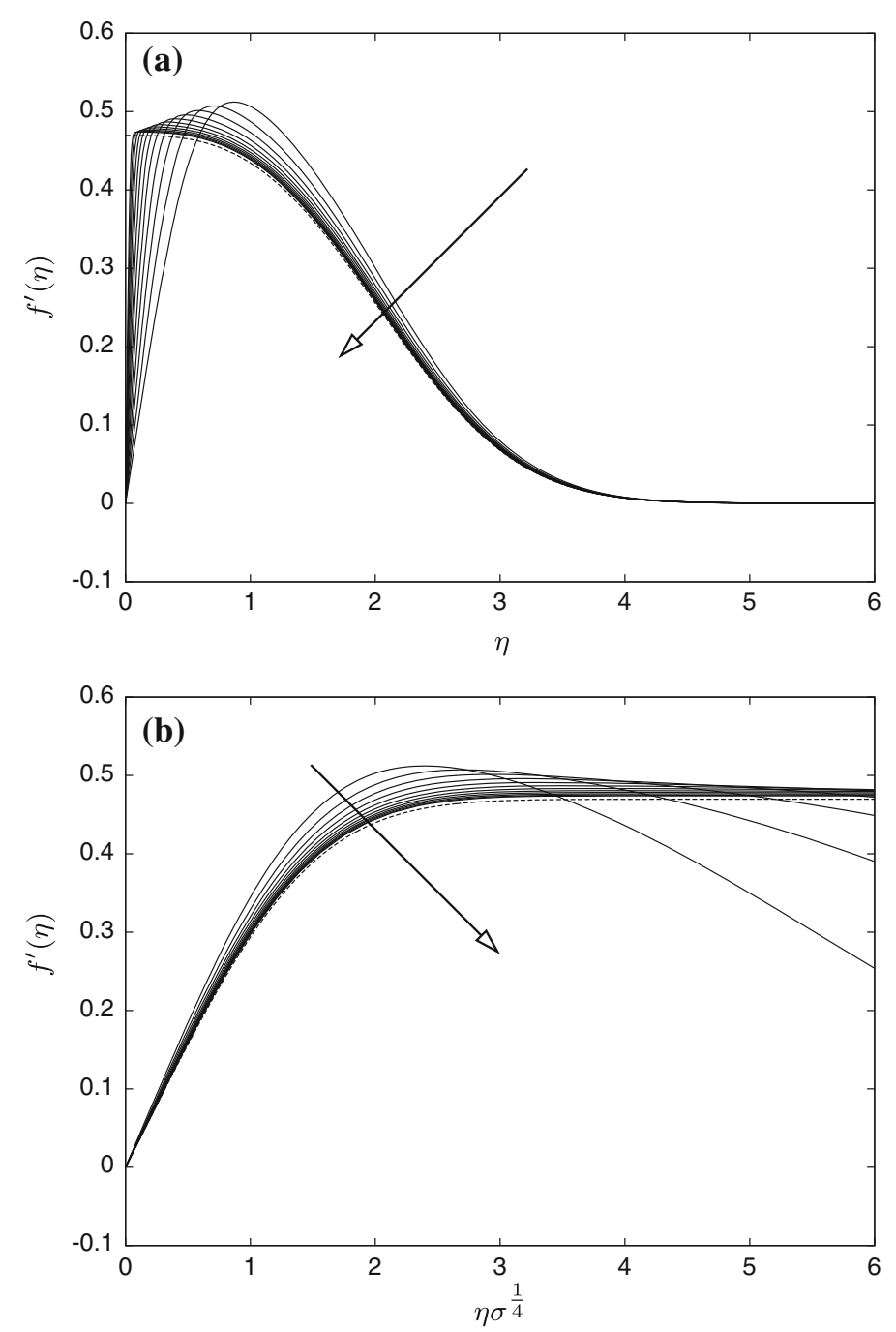

Fig. 11 A comparison between the asymptotic prediction for the behaviour of the outer (a) and inner (b) regions of the dominant eigenmode as $\zeta \rightarrow \zeta_{0}^{+} \zeta \zeta$ decreases in the direction of the arrow shown. Dashed lines show the leading-order asymptotic prediction and solid lines are the numerical results

first appearance of 'non-parallelism' in the subsequent corrections. Indeed, as expected, there are a number of similarities between this asymptotic description and the analysis of the short-scale eigenmode described above.

Clearly, the corrections in the outer flow do not satisfy the no-slip conditions $X_{\eta}=0$ on $\eta=0$, and an inner structure is therefore required. The inner layer is defined by the coordinate $\hat{\eta}=\eta /\left(\zeta_{0}-\zeta\right)^{1 / 4}=O(1)$, wherein the solution develops in the form

$$
\begin{aligned}
& X=\frac{1}{2}\left(\zeta_{0}-\zeta\right)^{\frac{1}{2}} F^{\prime \prime}(0) \hat{\eta}^{2}+\left(\zeta_{0}-\zeta\right) \hat{X}(\hat{\eta})+\cdots, \\
& G=\frac{1}{3}\left(\zeta_{0}-\zeta\right)^{\frac{3}{4}} \hat{\eta}^{3}+\left(\zeta_{0}-\zeta\right)^{\frac{5}{4}} \hat{G}(\hat{\eta})+\cdots .
\end{aligned}
$$

Note here that the leading-order terms are simply the Taylor expansions of the outer flow. This leads to the following system determining the correction terms $\hat{X}$ and $\hat{G}$ :

$$
\begin{aligned}
& \hat{X}_{\hat{\eta} \hat{\eta} \hat{\eta}}+F^{\prime \prime}(0)\left(\frac{5}{4} \hat{G}-\frac{1}{4} \hat{\eta} \hat{G}_{\hat{\eta}}\right)+\hat{\eta}^{2}\left(\frac{1}{4} \hat{\eta} \hat{X}_{\hat{\eta} \hat{\eta}}-\frac{3}{4} \hat{X}_{\hat{\eta}}\right)=0, \\
& \hat{G}_{\hat{\eta} \hat{\eta} \hat{\eta}}+2 \hat{\eta}\left(\frac{5}{4} \hat{G}-\frac{1}{4} \hat{\eta} \hat{G}_{\hat{\eta}}\right)+\hat{\eta}^{2}\left(\frac{1}{4} \hat{\eta} \hat{G}_{\hat{\eta} \hat{\eta}}-\hat{G}_{\hat{\eta}}\right)=\zeta_{0} F^{\prime \prime 2}(0) \hat{\eta}^{2} .
\end{aligned}
$$




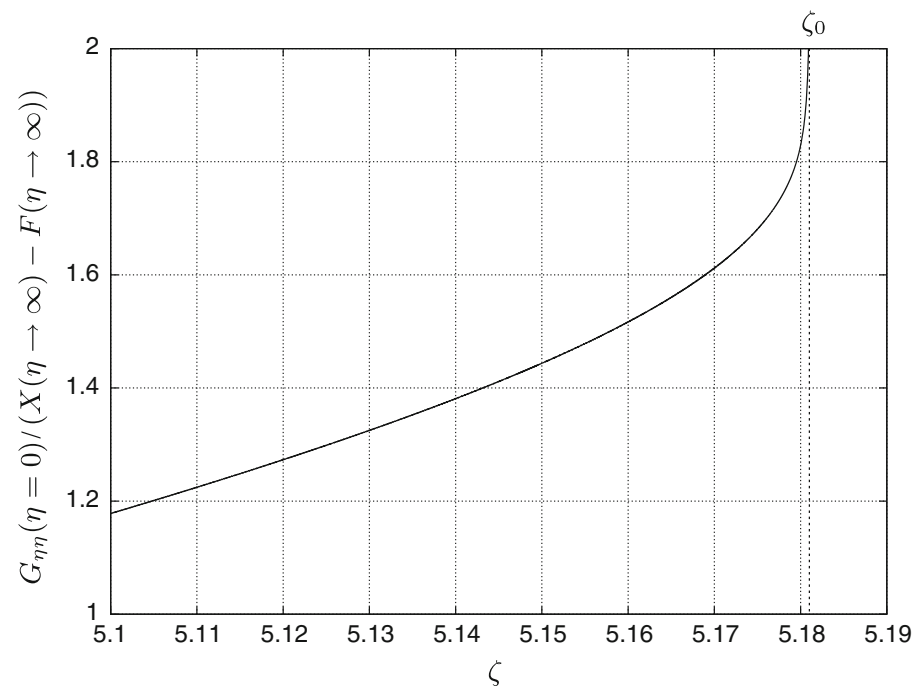

Fig. 12 The asymptotic description for $\zeta \rightarrow \zeta_{0}^{-}$predicts that $G_{\eta \eta}(\eta=0) /(X(\eta=0)-F(0)) \rightarrow 2$. Here, we show this comparison with the solutions of (9); the critical point $\zeta=\zeta_{0}$ is shown as the vertical (dashed) line

The appropriate solution for $\hat{G}$ is

$$
\hat{G}(\hat{\eta})=A_{0} \hat{\eta}^{2}+\frac{1}{60} \zeta_{0} F^{\prime \prime 2}(0) \hat{\eta}^{5}
$$

This leads to an inhomogeneous problem for $\hat{X}$ (which does not appear to have an analytic solution). Without determination of the amplitude $A_{0}$ or $\hat{X}$, we can nonetheless perform a comparison with the numerical results. Given the above structure, the prediction is that

$$
G_{\eta \eta}(\zeta, \eta=0) /(X(\zeta, \eta \rightarrow \infty)-F(\eta \rightarrow \infty)) \rightarrow 2, \quad \text { as } \zeta \rightarrow \zeta_{0}^{-},
$$

which is the comparison shown in Fig. 12, which appears to agree well with this result.

\subsection{The critical point $\zeta=\zeta_{1}$}

In Sect. 4.1, we demonstrated that an asymptotically short-scale eigenmode exists as $\zeta \rightarrow \zeta_{0}^{+}$when perturbing about the solution $X(\zeta, \eta)=F(\eta), Z(\zeta, \eta)=L(\eta)$. This mode exists because $\zeta_{0}$ is a singular point for which $\kappa_{\eta}\left(\zeta=\zeta_{0}, \eta\right)$ has a zero at $\eta=0$. The same analysis can equally be applied to $\zeta=\zeta_{1}^{+}$with minimal changes. For example, we replace the underlying base flow $F(\eta), L(\eta)$ with $X_{B}(\eta)=X\left(\zeta_{1}^{+}, \eta\right), Z_{B}(\eta)=Z\left(\zeta_{1}^{+}, \eta\right)$ and seek perturbations in the form

$$
(X, Z)=\left(X_{B}(\eta), Z_{B}(\eta)\right)+\delta(x(\eta), z(\eta)) E(\zeta),
$$

in the notation of (20).

The resulting 'parallel' flow eigenvalue problem for $\sigma$ is then analogous to (21), with $(F, L, f, l)$ replaced by $\left(X_{B}, Z_{B}, x, z\right)$. If, in addition, we include the homotopy parameter $\epsilon$ into the linearised problem, then it becomes a quadratic eigenvalue problem:

$$
\begin{aligned}
& x^{\prime \prime \prime}+\epsilon \sigma^{2} x^{\prime}+\sigma\left[(\zeta x+2 z) X_{B}^{\prime \prime}-\left(\zeta X_{B}^{\prime}+2 Z_{B}^{\prime}\right) x^{\prime}\right]=-x X_{B}^{\prime \prime}-x^{\prime \prime} X_{B}, \\
& z^{\prime \prime \prime}+\epsilon \sigma^{2} z^{\prime}+\sigma\left[(\zeta x+2 z) Z_{B}^{\prime \prime}-\left(\zeta X_{B}^{\prime}+2 Z_{B}^{\prime}\right) z^{\prime}\right]=-z^{\prime \prime} X_{B}-Z_{B}^{\prime \prime} x-X_{B}^{\prime} z^{\prime}-x^{\prime} Z_{B}^{\prime},
\end{aligned}
$$

with $x=x^{\prime}=z=z^{\prime}=0$ on $\eta=0$ and $x^{\prime}=z^{\prime}=0$ as $\eta \rightarrow \infty$. In this system, we have neglected derivatives of the base flow and eigenmode (with respect to $\zeta$ ) in the usual manner, recognising that the system is only 


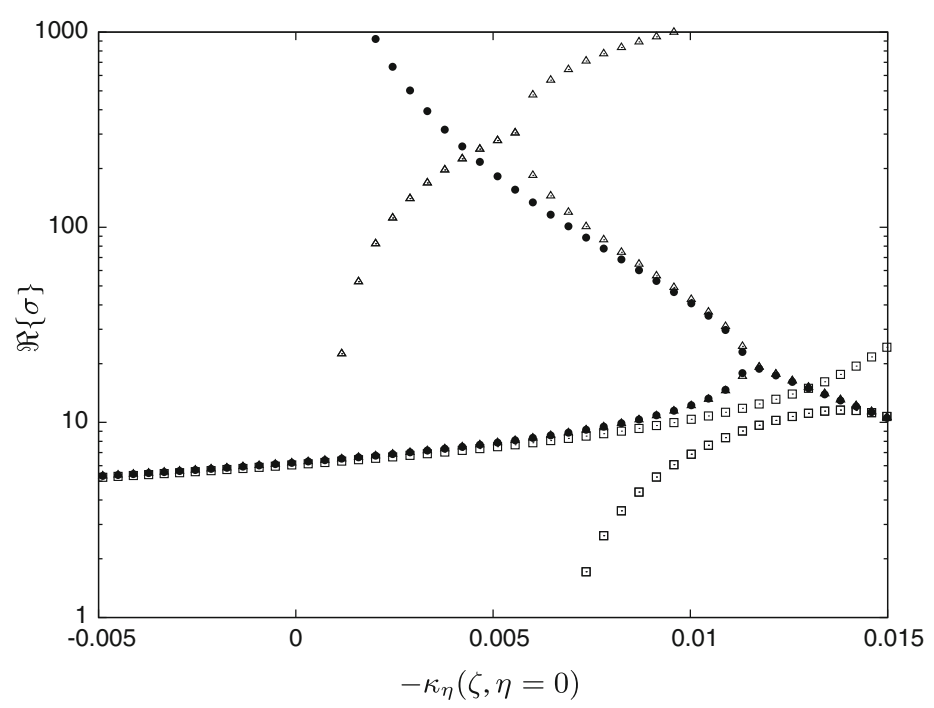

Fig. 13 A local 'parallel' eigenvalue analysis local to the critical point $\zeta_{1}$. The abscissa is taken to be $-\kappa_{\eta}(\zeta, \eta=0)$, as the critical point $\zeta_{1}$ is defined such that $-\kappa_{\eta}(\zeta, \eta=0)=0 ;-\kappa_{\eta}(\zeta, \eta=0)>0$ corresponds to $\zeta>\zeta_{1}$. The spectrum of modes is computed from the quadratic eigenvalue problem (including the homotopy parameter $\epsilon$ in both the base flow solution and the eigenmode). We show results for $\epsilon=5 \times 10^{-5}$ (squares), $10^{-6}$ (triangles). The filled circles denote the spectrum of modes for the $\epsilon \equiv 0$ solution as determined from a Crank-Nicolson scheme as outlined in Sect. 3.1.2

formally accurate in the short-scale $|\sigma| \gg 1$ limit. For the purposes of the numerical procedure, it is convenient to write the eigenproblem as

$$
\begin{gathered}
x^{\prime \prime \prime}+x X_{B}^{\prime \prime}+x^{\prime \prime} X_{B}=\sigma\left[\left(\zeta X_{B}^{\prime}+2 Z_{B}^{\prime}\right) x^{\prime}-\epsilon \hat{x}^{\prime}-(\zeta x+2 z) X_{B}^{\prime \prime}\right], \\
z^{\prime \prime \prime}+z^{\prime \prime} X_{B}+Z_{B}^{\prime \prime} x+X_{B}^{\prime} z^{\prime}+x^{\prime} Z_{B}^{\prime}=\sigma\left[\left(\zeta X_{B}^{\prime}+2 Z_{B}^{\prime}\right) z^{\prime}-\epsilon \hat{z}^{\prime}-(\zeta x+2 z) Z_{B}^{\prime \prime}\right] \\
\hat{x}=\sigma x, \\
\hat{z}=\sigma z,
\end{gathered}
$$

which is clearly a linear eigenvalue problem, but obtained at the expense of augmenting the system with the extra unknowns $\hat{x}, \hat{z}$ (which is standard practice used in solving polynomial eigenvalue problems). We discretise (40) by a second-order accurate scheme before evaluating the eigenvalue set $\{\sigma\}$ via a QZ algorithm.

In Fig. 13, we show the development of the real part of the eigenvalue $\sigma$, for $X_{B}, Z_{B}$ determined numerically by our global solver for a range of values of $\epsilon$. The corresponding $\epsilon \equiv 0$ eigenvalue is also shown, using the base flow determined from Crank-Nicolson marching, as described in Sect. 3.1.2. In this figure, we show $-\kappa_{\eta}(\zeta, \eta=0)$ as the abscissa, with $\zeta \rightarrow \zeta_{1}^{+}$corresponding to $-\kappa_{\eta}(\zeta, \eta=0) \rightarrow 0^{+}$. Our results show that, as expected, a short-scale eigenmode exists at $\zeta=\zeta_{1}$, where $\kappa_{\eta}(\eta=0)$ vanishes. However, these results demonstrate why some caution is required in the homotopy continuation, and why it is difficult to resolve the fine structure near $\zeta_{1}$ in the global numerical scheme (see Fig. 5). Even at $\epsilon=5 \times 10^{-5}$ (the data points shown as squares), the short-scale mode is clearly not present. At $\epsilon=10^{-6}$ (the data points shown as triangles), we resolve more of the spectrum of modes of the true $\epsilon \equiv 0$ solution (the data points shown as filled circles, as determined from Crank-Nicolson marching). However, for $\epsilon \equiv 0$ the short-scale mode (as $-\kappa_{\eta} \rightarrow 0^{+}$) is purely real, whereas for $\epsilon=10^{-6}$, the corresponding mode is quite different, since it becomes a complex conjugate pair when $-\kappa_{\eta}(\zeta, \eta=0) \approx 0.005$. This contamination of the short-scale spectrum of modes by the homotopy parameter makes accurate resolution of the correct behaviour very challenging in the global numerical solution even at small $\epsilon$ and can lead in some cases to spurious (oscillatory) behaviour in the neighbourhood of points where $\kappa$ changes sign.

Let us now turn to consider an asymptotic description in the neighbourhood of $\zeta=\zeta_{1}^{+}$, where the first sign change occurs in $\kappa_{\eta}(\eta=0)$. A plausible (double) asymptotic structure occurs, which for $\eta=O(1)$ has expansions

$$
\begin{aligned}
& X=X_{0}(\eta)+\left(\zeta-\zeta_{1}\right)^{\frac{1}{2}} A_{1}^{+} X_{0}^{\prime}(\eta)+\cdots \\
& G=G_{0}(\eta)+\left(\zeta-\zeta_{1}\right)^{\frac{1}{2}} A_{1}^{+} G_{0}^{\prime}(\eta)+\cdots
\end{aligned}
$$


(where $A_{1}^{+}$is a constant), whilst for $\hat{\eta}=\eta /\left(\zeta-\zeta_{1}\right)^{\frac{1}{4}}=O(1)$ :

$$
\begin{aligned}
& X=\frac{1}{2}\left(\zeta-\zeta_{1}\right)^{\frac{1}{2}} X_{0}^{\prime \prime}(0) \hat{\eta}^{2}+\left(\zeta-\zeta_{1}\right)^{\frac{3}{4}} \hat{X}(\hat{\eta})+\cdots \\
& G=\frac{1}{3}\left(\zeta-\zeta_{1}\right)^{\frac{3}{4}} \hat{\eta}^{3}+\left(\zeta-\zeta_{1}\right) A_{1}^{+} \hat{\eta}^{2}+\cdots
\end{aligned}
$$

This suggests that $\kappa(\eta=0)=O\left(\left(\zeta-\zeta_{1}\right)^{\frac{1}{2}}\right.$ as $\zeta \rightarrow \zeta_{1}^{+}$, which is in line with the Crank-Nicolson (marching) results in this limit; the structure as $\zeta \rightarrow \zeta_{1}^{-}$is similar, but with $\left(\zeta-\zeta_{1}\right)$ terms replaced by $\left(\zeta_{1}-\zeta\right)$ and the constant $A_{1}^{+}$replaced by another constant $A_{1}^{-}$.

4.4 The side-edge $\zeta=0^{+}$

Guided by the numerical results presented in Sect. 3, we first consider the nature of the flow on the plate, adjacent to the edge as $\zeta \rightarrow 0^{+}$. A 'meaningful' balance of terms suggests that in this region, we must have

$$
X=\zeta^{\frac{1}{2}} \hat{X}_{0}(\hat{\eta})+\zeta^{\frac{3}{4}} \hat{X}_{1}(\hat{\eta})+\cdots, \quad G=\zeta^{\frac{3}{4}} \hat{G}_{0}(\hat{\eta})+\zeta \hat{G}_{1}(\hat{\eta})+\cdots,
$$

where $\hat{\eta}=\eta / \zeta^{\frac{1}{4}}$.

Taking the leading terms (in powers of $\zeta$ ), we find

$$
\begin{aligned}
& \hat{X}_{0 \hat{\eta} \hat{\eta} \hat{\eta}}-\frac{3}{4} \hat{X}_{0 \hat{\eta} \hat{\eta}} \hat{G}_{0}+\frac{1}{4} \hat{X}_{0 \hat{\eta}} \hat{G}_{0 \hat{\eta}}=0, \\
& \hat{G}_{0 \hat{\eta} \hat{\eta} \hat{\eta}}+\frac{1}{2} \hat{G}_{0 \hat{\eta}}^{2}-\frac{3}{4} \hat{G}_{0 \hat{\eta} \hat{\eta}} \hat{G}_{0}=2 .
\end{aligned}
$$

There is an exact solution of the second equation, namely $\hat{G}_{0}=\frac{1}{3} \hat{\eta}^{3}$ (on the other hand, the solution for $\hat{X}_{0}$ is non-trivial). Further progress can be made with the $O(\zeta)$ term in the $G$ expansion, which is governed by the equation

$$
\hat{G}_{1 \hat{\eta} \hat{\eta} \hat{\eta}}-2 \hat{\eta} \hat{G}_{1}+\frac{5}{4} \hat{\eta}^{2} \hat{G}_{1 \hat{\eta}}-\frac{1}{4} \hat{\eta}^{2} \hat{G}_{1 \hat{\eta} \hat{\eta}}=0
$$

Usefully, this too has a trivial solution, namely

$$
\hat{G}_{1}=B \hat{\eta}^{2}
$$

where $B$ is a constant. The primary conclusion is therefore that $\kappa_{\eta}\left(\eta=0, \zeta \rightarrow 0^{+}\right)$and $G_{\eta \eta}\left(\eta=0, \zeta \rightarrow 0^{+}\right)$ are both $O\left(\zeta^{\frac{1}{2}}\right)$, which is in line, qualitatively, with our global numerical solution.

In the outer region where $\eta=O(1)$, (partly guided by our numerical results, partly from the information gleaned so far from the $\hat{\eta}=O$ (1) region), we expect

$$
\begin{aligned}
& X=X_{0}(\eta)+\zeta^{\frac{1}{2}} B X_{0}^{\prime}(\eta)+\cdots, \\
& G=G_{0}(\eta)+\zeta^{\frac{1}{2}} B G_{0}^{\prime}(\eta)+\cdots,
\end{aligned}
$$

where of course we have that $G_{0}^{\prime \prime}(0)=0$. We should emphasise (again) that there is no evidence of a sign change in $\kappa(\zeta=0, \eta)$, as $\eta$ varies, in contrast to the speculation in S61. 


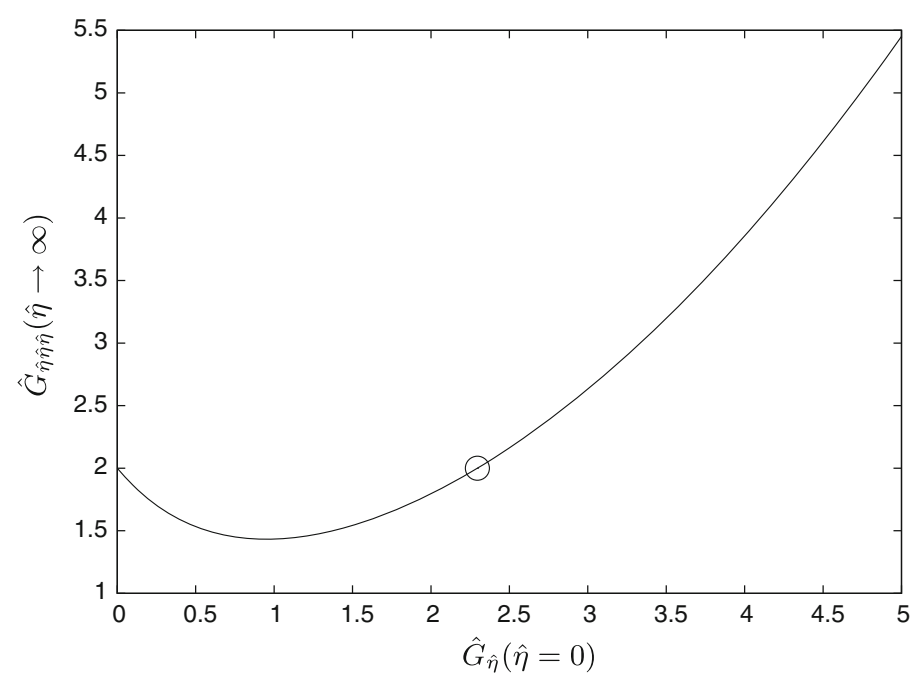

Fig. 14 Analysis as $\zeta \rightarrow 0^{-}$; the variation of $\hat{G}_{\hat{\eta} \hat{\eta} \hat{\eta}}(\hat{\eta} \rightarrow \infty)$ with $\hat{G}_{\hat{\eta}}(\hat{\eta}=0)$; the circle marks the second (non-trivial) solution

4.5 The side-edge $\zeta=0^{-}$

Let us now turn to a consideration of the limit $\zeta \rightarrow 0^{-}$. This too must have a singular structure, given (from our numerical results) that there must be a discontinuity in $X_{\eta \eta}(\eta=0)$ at $\zeta=0$. We develop an inner solution similar to that employed in the $\zeta \rightarrow 0^{+}$region (but note the key change in scaling of $G$ ):

$$
X=(-\zeta)^{\frac{1}{2}} \hat{X}(\hat{\eta})+\cdots, \quad G=(-\zeta)^{\frac{3}{4}} \hat{G}(\hat{\eta})+\cdots,
$$

where $\hat{\eta}=\eta /(-\zeta)^{\frac{1}{4}}$. Again, taking the leading terms (in powers of $\zeta$ ), we find

$$
\begin{aligned}
& \hat{X}_{\hat{\eta} \hat{\eta} \hat{\eta}}+\frac{3}{4} \hat{X}_{\hat{\eta} \hat{\eta}} \hat{G}+\frac{1}{4} \hat{X}_{\hat{\eta}} \hat{G}_{\hat{\eta}}=0, \\
& \hat{G}_{\hat{\eta} \hat{\eta} \hat{\eta}}-\frac{1}{2} \hat{G}_{\hat{\eta}}^{2}+\frac{3}{4} \hat{G}_{\hat{\eta} \hat{\eta}} \hat{G}=2 .
\end{aligned}
$$

The boundary conditions appropriate in this region are that

$$
\hat{X}(0)=\hat{X}_{\hat{\eta} \hat{\eta}}(0)=\hat{G}(0)=\hat{G}_{\hat{\eta} \hat{\eta}}(0)=0,
$$

together with $\hat{G} \sim \frac{1}{3} \hat{\eta}^{3}, \hat{X}_{\hat{\eta}} \sim \bar{\gamma}_{0} \hat{\eta}$ as $\hat{\eta} \rightarrow \infty$, where $\bar{\gamma}_{0}$ is a constant associated with the outer flow.

Again, the equation for $\hat{G}(\hat{\eta})$ does have an exact solution, namely $\hat{G}=\frac{1}{3} \hat{\eta}^{3}$, but it does not appear to play a role in this limit. Instead, we can find an alternative solution to the system (50)-(52) with $\hat{G}_{\hat{\eta}}(0) \neq 0$ and a standard matching process connects it to an outer $(\eta=O(1))$ flow (i.e. we have $\left.\hat{G}_{\hat{\eta} \hat{\eta} \hat{\eta}}(\infty)=2\right)$. Figure 14 shows the variation of $\hat{G}_{\hat{\eta} \hat{\eta} \hat{\eta}}(\hat{\eta} \rightarrow \infty)$ with $\hat{G}_{\hat{\eta}}(\hat{\eta}=0)$, and from this, it is clear that in addition to the trivial solution, a second (non-trivial) solution can be found with $\hat{G}_{\hat{\eta}}(\hat{\eta}=0) \approx 2.296$. It would appear that this non-trivial solution is the relevant one in this region.

The outer solution develops in the form (akin to (48a) and (48b)):

$$
\begin{aligned}
& X=X_{0}(\eta)+(-\zeta)^{\frac{1}{4}} C X_{0}^{\prime}(\eta)+\cdots, \\
& G=G_{0}(\eta)+(-\zeta)^{\frac{1}{4}} C G_{0}^{\prime}(\eta)+\cdots,
\end{aligned}
$$

where $C$ is a constant, $X_{0}(\eta) \sim \frac{1}{2} \bar{\gamma}_{0} \eta^{2}, G_{0}(\eta) \sim \frac{1}{3} \eta^{3}$ as $\eta \rightarrow 0$.

These scalings suggest that $G_{\eta} /\left(\zeta \rightarrow 0^{-}, \eta=0\right)=-\kappa\left(\zeta \rightarrow 0^{-}, \eta=0\right) \sim 2.296(-\zeta)^{\frac{1}{2}}$ on approaching the side edge from the off-plate direction; Fig. 15 shows good evidence of this as we decrease the homotopy parameter $\epsilon$. 


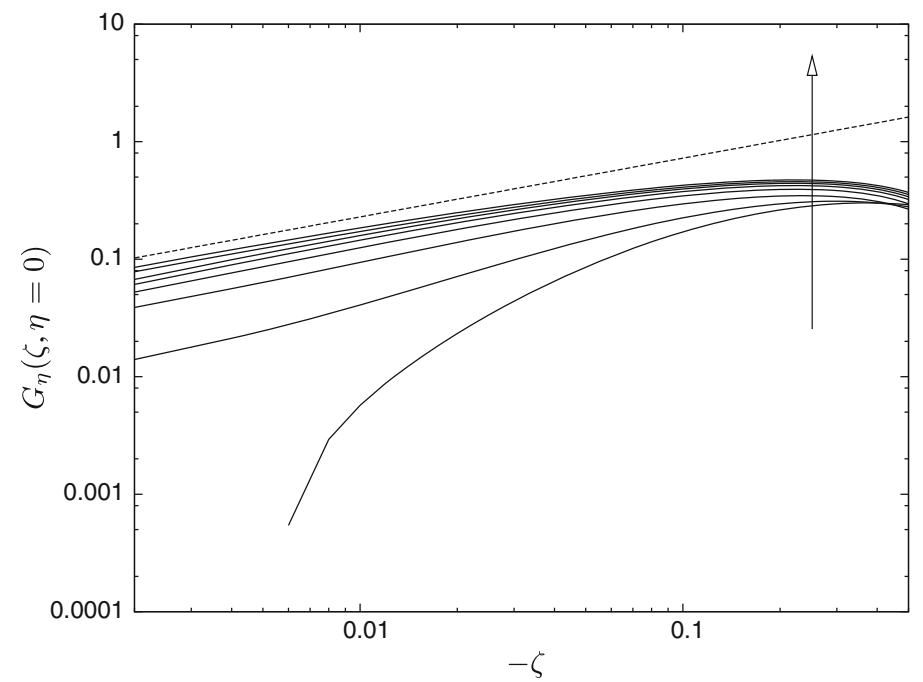

Fig. 15 The asymptotic description for $\zeta \rightarrow 0^{-}$predicts that $G_{\eta} \sim 2.296(-\zeta)^{\frac{1}{2}}$ when evaluated at $\eta=0$. Here, we show this comparison for $\epsilon=32 \times 10^{-4}, 16 \times 10^{-4}, 8 \times 10^{-4}, 4 \times 10^{-4}, 2 \times 10^{-4}, 10^{-4}, 5 \times 10^{-5}, 10^{-5}$, with $\epsilon$ decreasing in the direction of the arrow shown; the asymptotic prediction is shown as the dashed line

4.6 The critical point $\zeta=\bar{\zeta}_{0}^{-}$

The zone close to $\bar{\zeta}_{0}$ is clearly significant, since it appears to be the region where non-uniqueness stems from. Our numerical results suggest that $\bar{\zeta}_{0} \approx-0.95$, and we expect that for $\zeta=\bar{\zeta}_{0}^{+}, G \sim \mu_{0} \eta^{3}$ and $X \sim \mu_{1} \eta$ as $\eta \rightarrow 0$ for constants $\mu_{0,1}$.

We proceed by defining the following quantities close to $\zeta=\bar{\zeta}_{0}^{-}$:

$$
\begin{aligned}
G & =\left(\bar{\zeta}_{0}-\zeta\right)^{\frac{3}{4}} \hat{G}(\hat{\eta}), \\
X & =\left(\bar{\zeta}_{0}-\zeta\right)^{\frac{1}{4}} \hat{X}(\hat{\eta}), \\
\hat{\eta} & =\eta /\left(\bar{\zeta}_{0}-\zeta\right)^{\frac{1}{4}}
\end{aligned}
$$

Therefore, to leading order, we obtain

$$
\begin{aligned}
& \hat{X}_{\hat{\eta} \hat{\eta} \hat{\eta}}+\frac{3}{4} \hat{G} \hat{X}_{\hat{\eta} \hat{\eta}}=0, \\
& \hat{G}_{\hat{\eta} \hat{\eta} \hat{\eta}}+\frac{3}{4} \hat{G} \hat{G}_{\hat{\eta} \hat{\eta}}-\frac{1}{2} \hat{G}_{\hat{\eta}}^{2}=2+\bar{\zeta}_{0} \hat{X}_{\hat{\eta}}^{2},
\end{aligned}
$$

subject to

$$
\begin{aligned}
& \hat{X}(0)=\hat{X}_{\hat{\eta} \hat{\eta}}(0)=\hat{G}(0)=\hat{G}_{\hat{\eta} \hat{\eta}}(0)=0, \\
& \hat{X} \sim \mu_{1} \hat{\eta}, \quad \hat{G} \sim \mu_{0} \hat{\eta}^{3}, \quad \text { as } \quad \hat{\eta} \rightarrow \infty,
\end{aligned}
$$

where it is easy to show that $\mu_{0}=\left(2+\bar{\zeta}_{0} \mu_{1}^{2}\right) / 6$.

The only appropriate solution for $\hat{X}$ appears to be the trivial one, i.e. $\hat{X}=\mu_{1} \hat{\eta}$. If we then write $\hat{G}=$ $\left(3 \mu_{0}\right)^{\frac{1}{4}} G^{*}, \hat{\eta}=\left(3 \mu_{0}\right)^{-\frac{1}{4}} \eta^{*}$, then the resulting system is again (51). However, in this case, it appears that both the trivial and non-trivial solutions for $G^{*}$ are relevant here, and so this is important confirmation of the nonuniqueness observed in our full numerical solutions. It is our assertion that the trivial solution $\left(G^{*}=\frac{1}{3} \eta^{* 3}\right)$ corresponds to the $\lambda \approx 0.51$ solution, with smooth behaviour in the vicinity of $\zeta=\bar{\zeta}_{0}$, whilst the non-trivial solution corresponds to $\lambda=0$, which leads to a discontinuity in $\zeta$ derivatives, as observed in Fig. 9. 


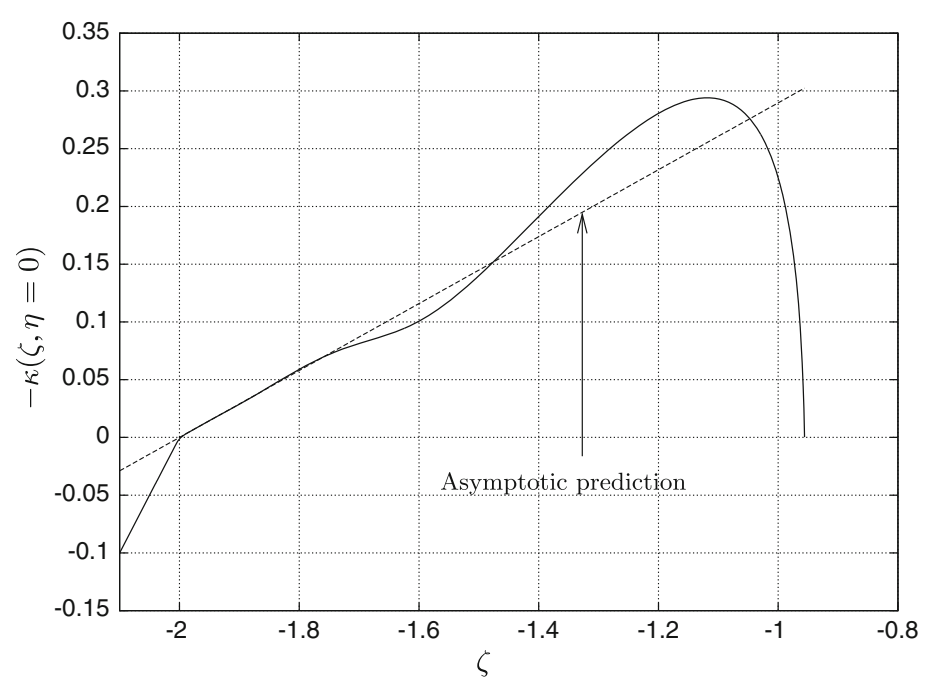

Fig. 16 The distribution of $\kappa$ along $\eta=0$ for the $\lambda=0$ solution over the range $\zeta \in\left[-2.1, \bar{\zeta}_{0}\right]$. The (as $\zeta \rightarrow-2^{+}$) asymptotic prediction of Sect. 4.7, $\kappa(\zeta, \eta=0) \sim 0.28972(\zeta+2)$, is shown as the dashed line. Note that $\kappa \equiv-G_{\eta}$ and are insensitive to $\epsilon$ for $\epsilon \ll 1$

4.7 Off the plate: $\zeta \rightarrow-2(\lambda=0)$

In the case of $\lambda \approx 0.51$, the solution in this region appears to be entirely smooth, and as a result the asymptotic structure is relatively benign. The picture for the $\lambda=0$ solution is more subtle though; in the limit of $\zeta \rightarrow-2^{+}$, the solution develops in the form

$$
\begin{aligned}
& X=\eta+(\zeta+2) \tilde{X}(\eta)+\cdots \\
& G=(\zeta+2) \tilde{G}(\eta)+\cdots
\end{aligned}
$$

Although this is consistent with S61, the system governing $\tilde{X}(\eta)$ and $\tilde{G}(\eta)$ differs from that stated in S61 which we believe to be in error; instead, the problem is more akin to that in the neighbourhood of a stagnation point flow and as such is non-linear (S61 proposed a linear form), specifically

$$
\begin{aligned}
& \tilde{X}_{\eta \eta \eta}+2 \eta \tilde{X}_{\eta \eta}-\tilde{X}_{\eta \eta} \tilde{G}+\tilde{X}_{\eta} \tilde{G}_{\eta}=0, \\
& \tilde{G}_{\eta \eta \eta}-\tilde{G} \tilde{G}_{\eta \eta}+2 \eta \tilde{G}_{\eta \eta}+\tilde{G}_{\eta}^{2}+4 \tilde{X}_{\eta}-1=0,
\end{aligned}
$$

subject to $\tilde{X}(0)=\tilde{X}_{\eta \eta}(0)=\tilde{G}(0)=\tilde{G}_{\eta \eta}(0)=0$, together with $\tilde{G}_{\eta}(\eta \rightarrow \infty) \rightarrow 1, \tilde{X}_{\eta}(\eta \rightarrow \infty) \rightarrow 0$.

The appropriate solution of (64) appears to be $\tilde{X} \equiv 0$, which is consistent with our numerical results, which show little evidence of singular behaviour in $X_{\eta}(\eta=0)$ in this region. The reduced equation (65) then admits a continuous spectrum of solutions in the range $-1<\tilde{G}_{\eta}(0)<1$, which take the form (as $\eta \rightarrow \infty$ ):

$$
\tilde{G} \rightarrow \eta+C_{0}+\frac{C_{1}}{\eta}+\cdots ;
$$

however, a detailed investigation into this system reveals that for $\tilde{G}_{\eta}(\eta=0) \approx 0.28972, \tilde{G}_{\eta} \rightarrow 1$ exponentially; it appears that it is this solution that is appropriate in the global problem. In Fig. 16, we show a comparison between the asymptotic prediction and the global solution for the $\lambda=0$ state in the range $\zeta \in\left[-2.1, \bar{\zeta}_{0}\right]$, indicating excellent agreement. The conclusion, therefore, is that there is a discontinuity in $\kappa_{\zeta}$ at $\zeta=-2$ in this case.

\section{Discussion}

In this work, we have revisited the quarter-infinite plate problem first introduced by S61 and [5]. The governing equations for the flow in a near-edge region are diffusive in the coordinate normal to the plate but parabolic in 
the crossflow direction. The system, although parabolic, is bidirectional with both of the boundary conditions far onto the plate and far off the plate corresponding to 'inflow' boundaries.

The numerical task of solving for the full flow field remains a challenging one today, and it is hardly surprising that the system remained unsolved in 1961. Because of the uncertainty of the location of the critical locations a priori, we did not resort to non-uniform meshing and hence were solving for up to two million degrees of freedom to accurately resolve the many important areas of the flow, including the discontinuity at the plate edge and the other critical points. This large data set necessitates the use of sparse solvers to restrict the memory footprint during the linear solver phase. The authors also performed a number of computational experiments with the 'original', $\epsilon \equiv 0$, system (9). A range of different differencing schemes were investigated to treat the bidirectional nature of the flow on and off the plate. However, a key problem is that the regions of bidirectionality must be determined as part of the solution and methods that employed simple windward differencing were not effective. Our conclusion is that the discontinuities of $\zeta$ derivatives, coupled with the bidirectional nature of the parabolicity, combine to make even global solution of the $\epsilon \equiv 0$ system highly challenging and perhaps even unfeasible. Moreover, local solution methods applied to the $\epsilon \equiv 0$ system were entirely without merit.

Our decision to investigate the $\epsilon \rightarrow 0$ limit was, in large part, motivated by the lack of effectiveness of all other methods that were employed. Whilst this homotopy method proves to be surprisingly effective, it is not an approach that can be used blindly. We have made a significant effort to make comparisons with asymptotic descriptions of critical regions and also to validate the global $(\epsilon \rightarrow 0)$ solution with $(\epsilon \equiv 0)$ purely parabolic marching approaches in regimes where the direction of information propagation is unidirectional. The choice of how to make the system elliptic is entirely arbitrary; for the majority of our investigation, we included the extra terms $\left(\epsilon X_{\eta \zeta \zeta}, \epsilon Z_{\eta \zeta \zeta}\right)$ into the $(X, Z)$ equations (9). However, for additional validation, we have also considered $\left(\epsilon X_{\zeta \zeta}, \epsilon Z_{\zeta \zeta}\right)$, which results in precisely the same behaviour as $\epsilon \rightarrow 0$.

In the absence of any numerical results, S61 was forced to speculate regarding the features of the flow. We have shown in this work that the true features of the global flow field are in fact somewhat more complicated than one might infer from S61. In particular, we have shown:

(1) The condition imposed by S61 as $\zeta \rightarrow-\infty$ is one of two possibilities. In our notation here, S61's far-field flow corresponds to $\lambda=0$, but another possibility exists with $\lambda \neq 0$. Although the $\lambda=0$ state can be found (in agreement with the far-field off-plate flow assumed in S61), $Z_{\zeta}$ appears to have a discontinuity at a critical off-plate location $\zeta=\bar{\zeta}_{0}$; the more benign $\lambda \approx 0.51$ state we present herein may be viewed as more plausible in this sense.

(2) S61 suggested that the most likely scenario is that $\kappa$ (indicating the direction of disturbance propagation in the $\zeta$ coordinate) has a sign change at some $\eta$ on the side-edge $\zeta=0$. However, this is not the case; the solution is again more complicated with the region in which $\kappa$ changes sign terminating precisely at the edge of the plate, $\zeta=0, \eta=0$.

(3) The bidirectional region on the plate exists in the finite range $\zeta \in\left[0, \zeta_{1}\right]$. The (natural) assumption made in S61 was that this region extended all the way to $\zeta=\zeta_{0}>\zeta_{1}$, which is not quite the case. The critical location $\zeta_{0}$ is associated instead with a discontinuity in $X_{\zeta}$ and $Z_{\zeta}$, but no local sign change in $\kappa$.

S61's interest in this problem was perhaps driven in part by his work $[11,14]$ on the impulsively started semi-infinite flat plate. At a finite time after the impulsive motion has begun, the flow immediately behind the leading edge is a spatially varying Blasius solution, but the flow far from the edge is a parallel Rayleigh layer. Stewartson's contention was that the spatially developing Blasius solution (with its non-zero streamwise derivatives) can only connect to the Rayleigh layer solution (for which all streamwise derivatives are zero) if they are separated by an essential singularity. Stewartson proposed that this singularity exists at a distance $U t$ from the leading edge, where $U$ is the plate speed and $t$ the time since the plate was placed in motion. Similar features exist in this problem, as has been noted both in S61 and herein. In our asymptotic descriptions, we have allowed for discontinuous $\zeta$ gradients; ${ }^{1}$ however, we cannot rule out that essential singularities have some role to play in smoothing such discontinuities over sufficiently small $\zeta$ scales. It should be noted that instances of discontinuous derivatives have been found in the past in boundary-layer flows, for example in marginal separation $[9,16]$. Interestingly, Ruban and Vonatsos [10] recently presented (unsteady) boundary-layer results that go further than merely discontinuous derivatives, presenting evidence of discontinuous solutions. Nevertheless, S61 suggests (somewhat speculatively) that an essential singularity exists at

\footnotetext{
${ }^{1}$ We note that the discussion on page 9 of S61 also implies discontinuous derivatives at $\zeta=-2$, although the governing equations presented in that discussion are actually in error.
} 

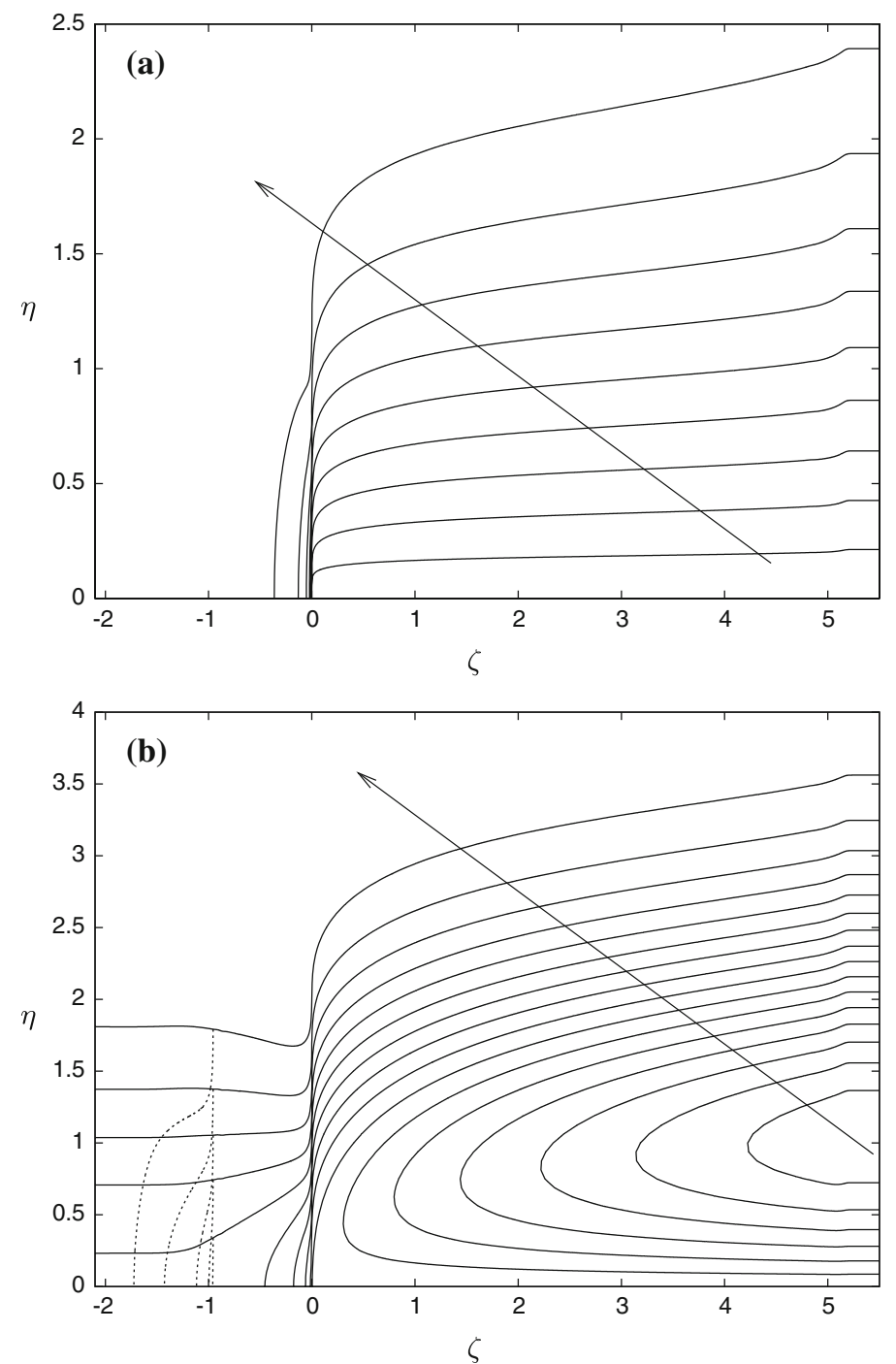

Fig. 17 Contours of $\mathbf{a} X_{\eta}(\zeta, \eta)$ (corresponding to the streamwise velocity) and $\mathbf{b} Z_{\eta}(\zeta, \eta)$ (corresponding to the crossflow velocity) for the $\lambda \approx 0.51$ solution. In $\mathbf{b}$, the $\lambda=0$ solution corresponds to the dashed lines (rather than the solid lines) for $\zeta<\bar{\zeta}_{0} \approx-0.95$. Contours are (a) $0.1,0.2, \ldots, 0.9$ and (b) $-0.6,-0.5, \ldots, 0.9$, increasing in the direction of the arrow. These contours are insensitive to the value of $\epsilon$ for $\epsilon \ll 1$

$\zeta=\zeta_{0}$, and our local short-scale eigenmode analysis also indicates a singular behaviour for linearised perturbations as $\zeta \rightarrow \zeta_{0}^{+}$, as described by (22). Our results also indicate that this same short-scale eigenmode provides the crucial mechanism for perturbing the $X=F, Z=L$ solution (in a singular fashion, as described in Sect. 4.2). However, a note of caution should be added, in that any numerical discretisation scheme that relies on the existence of a Taylor series will inevitably struggle if an essential singularity is indeed present.

Finally, in Fig. 17, we try to provide a more physical view of the full flow field for (a) the streamwise velocity $X_{\eta}$ and (b) the crossflow velocity $Z_{\eta}$; note that these quantities correspond to $u$ and $w$ via the rescaling of (8), which depends upon the local Reynolds number. In Fig. 17a, we show the contours of constant streamwise velocity for the $\lambda \approx 0.51$ solution; there is very little difference in $u$ between the two possible solutions and therefore we choose to present only one of them. In Fig. 17b, we show the corresponding contours of constant crossflow, $w$, velocity for both $\lambda \approx 0.51$ and $\lambda=0$. For the $\lambda=0$ state, the contours are the dashed lines for $\zeta<\bar{\zeta}_{0}$; the two states are the same for $\zeta<\bar{\zeta}_{0}$.

The non-uniqueness observed for values of $\zeta<\bar{\zeta}_{0}$ is an unusual feature but is supported by the asymptotic analysis of Sect. 4.6 and (importantly) is associated with a singular point in the parabolic system. Although the streamwise velocity $\left(X_{\eta}\right)$ shows little quantitative change between the two states in this regime, there is a 
significant difference in the crossflow velocity $\left(Z_{\eta}\right)$. In the case of $\lambda \approx 0.51$, the crossflow develops smoothly in the neighbourhood of $\zeta=\zeta_{0}$ (and also close to $\zeta=-2$ ); indeed, it is only weakly dependent on $\zeta$ for $\zeta<\bar{\zeta}_{0}$. In the case of $\lambda=0$, in addition to the apparent discontinuity in $Z_{\zeta}$ at $\zeta=\bar{\zeta}_{0}$, a further discontinuity in the $\zeta$ derivative occurs at $\zeta=-2$ (as suggested in S61). One might justifiably view (based on physical intuition alone) the occurrence of these two discontinuities in $\zeta$ gradients (in the off-plate flow) as being the less likely of the two solutions.

\section{References}

1. Blasius, H.: Flüssigkeiten mit keinen Reibung. Z. Math. Phys. 56, 1-37 (1908)

2. Davis, R.T.: Laminar incompressible flow past a semi-infinite flat plate. J. Fluid Mech. 27, 691-704 (1967)

3. Duck, P.W.: Laminar flow over unsteady humps: the formation of waves. J. Fluid Mech. 160, 465-498 (1985)

4. Glauert, M.B., Lighthill, M.J.: The axisymmetric boundary layer on a long thin cylinder. Proc. R. Soc. Lond. A 230, 188-203 (1955)

5. Howarth, L., Stewartson, K.: On the flow past a quarter-infinite plate using Oseen's equations. J. Fluid Mech. 7, 1-21 (1960)

6. Messiter, A.F.: Boundary-layer flow near the trailing edge of a flat plate. SIAM J. Appl. Math. 18, 241-257 (1970)

7. Neiland, V.Y.: Theory of laminar boundary layer separation in supersonic flow. Fluid Dyn. 4, 33-35 (1969)

8. Phillips, W.R.C.: On a class of unsteady boundary layers of finite extent. J. Fluid Mech. 319, 151-170 (1996)

9. Ruban, A.I.: Singular solution of boundary layer equations which can be extended continuously through the point of zero surface friction. Izv. Akad. Nauk SSSR: Mekh. Zhidk. Gaza 6, 4252 (1981) (Engl. transl. Fluid Dyn. 16(6),835843)

10. Ruban, A.I., Vonatsos, K.N.: Discontinuous solutions of the boundary-layer equations. J. Fluid Mech. 614, 407-424 (2008)

11. Stewartson, K.: On the impulsive motion of a flat plate in a viscous fluid. Quart. J. Mech. Appl. Math. 4(2), 182-198 (1951)

12. Stewartson, K.: Viscous flow past a quarter-infinite plate. J. Aero. Sci. 28, 1-10 (1961)

13. Stewartson, K.: On the flow near the trailing edge of a flat plate. Proc. R. Soc. Lond. A 306, 275-290 (1968)

14. Stewartson, K.: On the impulsive motion of a flat plate in a viscous fluid II. Quart. J. Mech. Appl. Math. 26(2), 143-152 (1973)

15. Stewartson, K.: Multistructured boundary layers on plates and related bodies. Adv. Appl. Mech. 14, 145-239 (1974)

16. Stewartson, K., Smith, F.T., Kaups, K.: Marginal separation. Stud. Appl. Math. 67, 4561 (1982)

17. van Dyke, M.: Perturbation Methods in Fluid Mechanics. Parabolic Press, Stanford (1975) 